\title{
AN EXTENSION OF THE CROUZEIX-RAVIART SPACE TO GENERAL MESHES WITH APPLICATION TO QUASI-INCOMPRESSIBLE LINEAR ELASTICITY AND STOKES FLOW
}

\author{
DANIELE A. DI PIETRO AND SIMON LEMAIRE
}

\begin{abstract}
In this work we introduce a discrete functional space on general polygonal or polyhedral meshes which mimics two important properties of the standard Crouzeix-Raviart space, namely the continuity of mean values at interfaces and the existence of an interpolator which preserves the mean value of the gradient inside each element. The construction borrows ideas from both Cell Centered Galerkin and Hybrid Finite Volume methods. The discrete function space is defined from cell and face unknowns by introducing a suitable piecewise affine reconstruction on a (fictitious) pyramidal subdivision of the original mesh. Two applications are considered in which the discrete space plays an important role, namely (i) the design of a locking-free primal (as opposed to mixed) method for quasi-incompressible planar elasticity on general polygonal meshes; (ii) the design of an inf-sup stable method for the Stokes equations on general polygonal or polyhedral meshes. In this context, we also propose a general modification, applicable to any suitable discretization, which guarantees that the velocity approximation is unaffected by the presence of large irrotational body forces provided a Helmholtz decomposition of the right-hand side is available. The relation between the proposed methods and classical finite volume and finite element schemes on standard meshes is investigated. Finally, similar ideas are exploited to mimic key properties of the lowest-order Raviart-Thomas space on general polygonal or polyhedral meshes.
\end{abstract}

\section{INTRODUCTION}

In the context of industrial simulators, lowest-order methods for diffusive problems on general polygonal or polyhedral meshes have received increasing attention over the last few years. The reasons are multifold. Using general polyhedral elements may ease the discretization of complex domains, and disposing of discretization methods applicable to general meshes is mandatory whenever the user cannot adapt the mesh to the needs of the numerical scheme. This is the case, e.g., in the context of computational geosciences, where the discretization of the subsoil is developed in a separate stage, and is focused on integrating physical and geometric data resulting from the seismic analysis. Fairly general meshes can thus be encountered, featuring, e.g., nonmatching interfaces corresponding to geological faults or general polyhedral elements resulting from the degeneration of hexahedral cells in eroded layers. Polyhedral elements may additionally be present in near wellbore regions, where the use of radial meshes can be prompted by (qualitative) a priori

Received by the editor November 19, 2012 and, in revised form, June 5, 2013.

2010 Mathematics Subject Classification. Primary 65N08, 65N30; Secondary 74B05, 76D07. 
knowledge of the solution. Nonconforming $h$-refinement can also appear at specific locations where the resolution needs to be increased, or when moving fronts are present. In this context, the use of lowest-order methods is justified both by the inherent uncertainty associated to physical data and the need to keep computational costs within affordable bounds.

Among the methods that have appeared in recent years, the most directly related to the present work are the Mimetic Finite Difference (MFD) method of Brezzi, Lipnikov et al. [11 13, the Hybrid Finite Volume (HFV) method of Eymard, Gallouët and Herbin [25], and the Mixed Finite Volume (MFV) method of Droniou and Eymard [20]. The close relation among these methods has recently been investigated in [22]. The main result of the present work is the construction of a discrete space of piecewise affine functions which extends two important properties of the classical Crouzeix-Raviart space [15] to general meshes, namely,

(CR1) the continuity of mean values at interfaces. Since we deal with piecewise affine functions, this property is equivalent to the continuity at face barycenters;

(CR2) the existence of an interpolator which preserves the mean value of the gradient inside each element. Such interpolator can play the role of a Fortin operator [10].

Let $\mathcal{K}_{h}$ denote a general polygonal or polyhedral mesh of the bounded domain $\Omega$ matching the regularity requirements discussed in Section 2 In the spirit of Cell Centered Galerkin (ccG) methods [16, the discrete space is constructed in three steps:

(i) We fix the vector space $\mathbb{V}_{h}$ of face- and cell-centered degrees of freedom (DOFs) on $\mathcal{K}_{h}$.

(ii) We define a discrete gradient reconstruction operator $\mathfrak{G}_{h}$ acting on $\mathbb{V}_{h}$. The reconstructed gradient is piecewise constant on a fictitious pyramidal submesh $\mathcal{P}_{h}$ obtained by subdividing each element using one interior point (the cell center), and it results from the sum of two terms: a consistent part depending on face unknowns only, plus a subgrid correction involving both face and cell unknowns. The continuity of mean values at interfaces is ensured by finely tuning the latter contribution.

(iii) We define an affine reconstruction operator $\mathfrak{R}_{h}$ acting on $\mathbb{V}_{h}$ which maps every vector of DOFs on a broken affine function on $\mathcal{P}_{h}$. This function is obtained by perturbing the (unique) face unknown associated to each pyramid with a linear correction based on the discrete gradient $\mathfrak{G}_{h}$. The discrete space is then defined as

$$
\mathfrak{C} \Re\left(\mathcal{K}_{h}\right):=\Re_{h}\left(\mathbb{V}_{h}\right) \subset \mathbb{P}_{d}^{1}\left(\mathcal{P}_{h}\right),
$$

with $\mathbb{P}_{d}^{1}\left(\mathcal{P}_{h}\right)$ space of broken affine functions on $\mathcal{P}_{h}$; cf. (4).

The pyramidal submesh is fictitious in the sense that all the relevant geometric information can be computed on the primal mesh, which is therefore the only one that needs to be described and manipulated by the end-user. In Appendix A, similar ideas are used to construct a $\boldsymbol{H}(\operatorname{div} ; \Omega)$-conforming discrete space on general meshes which mimics two key properties of the standard lowest-order RaviartThomas space, namely the (full) continuity of normal values at interfaces and the approximation of vector-valued fields. We mention at this point the recent work of Vohralík and Wohlmuth [34,35] which proposes efficient implementation strategies 
for classical nonconforming and mixed finite element approximations of diffusive problems, and addresses general meshes with a different approach.

The first application we consider is linear elasticity, which was also the original motivation for this work. Our main result is the design of a primal (as opposed to mixed) method based on the space $\mathfrak{C} \mathfrak{R}\left(\mathcal{K}_{h}\right)$ (and thus applicable to general meshes) which is locking-free in the quasi-incompressible limit in two space dimensions (the required regularity estimate for the continuous solution is still an open problem in higher space dimensions).

It has been long known that the accuracy of lowest-order $H^{1}$-conforming (Lagrange) finite element approximations deteriorates when quasi-incompressible materials are considered, i.e., for large values of the first Lamé parameter $\lambda$; cf., e.g., 8, Section 11.3]. This is a consequence of the fact that the convergence estimates are not uniform in $\lambda$, which, in turn, reflects the inability of the discretization space to accurately represent nontrivial solenoidal fields. One classical way of circumventing this problem is the use of mixed formulations, resulting in methods which converge uniformly in $\lambda$, but which are often computationally more expensive than primal methods. Examples include the PEERS method of Arnold, Brezzi and Douglas [2], the mixed method of Stenberg [31], and the mixed methods of Chavan, Lamichhane and Wohlmuth [14, and Lamichhane and Stephan 30. All these methods require matching triangular or quadrilateral meshes. General meshes similar to the ones addressed in this work have been recently considered by Beirão da Veiga [3], who introduces a mixed MFD method which does not lock in the quasi-incompressible limit.

The problem of locking has also been addressed without resorting to mixed formulations, and several methods can be found in the literature. On matching triangular meshes, we can cite, e.g., the $p$-version method of Vogelius [33, and the nonconforming methods of Falk [26]. Concerning general meshes, we mention the very recent work of Beirão da Veiga, Brezzi, and Marini [4] on virtual elements for linear elasticity. In the MFD framework, we cite the method for the twodimensional Stokes problem proposed by Beirão da Veiga, Gyrya et al. [5], whose analysis and extension to three space dimensions have been considered in [6]. In both cases the authors consider a (possibly fourth-order) tensor viscosity, which allows an extension to linear elasticity. We note, moreover, that inf-sup stable mixed methods for the Stokes problem can be, whenever the pressure is discontinuous, converted to a locking-free primal method for the pure displacement linear elasticity problem by static condensation of pressures. This strategy can be applied to the method of [5, 6]. In the MFV framework, we can cite the work of Droniou and Eymard [21, where the Stokes problem is also addressed. In this work we take inspiration from the classical Crouzeix-Raviart method of Brenner and Sung [9]. Here, while property (CR1) ensures adequate conformity properties to the space, property (CR2) plays an important role in deriving an error estimate uniform in $\lambda$. Another source of inspiration is the work of Hansbo and Larson [28, 29, where a locking-free Discontinuous Galerkin $(\mathrm{dG})$ method on matching triangular meshes is analyzed; see also Di Pietro and Nicaise [19] for an extension to composite materials. Coercivity is here ensured by a least-square penalization of interface jumps. In our method, a similar device is required in the case of mixed-type boundary conditions to invoke a discrete Korn's inequality on broken polynomial spaces [7]. For the pure displacement problem, the naturally coercive Navier-Cauchy formulation allows us 
to circumvent the use of Korn's inequality. Up to a suitable treatment of the right-hand side, it is possible to prove for our scheme a local conservation property similar to finite volume methods. In this case, the face unknowns act as Lagrange multipliers associated to the flux continuity constraint. Yet another treatment of the right-hand side allows us to recover the method of [9] on matching simplicial meshes.

As a second, closely related application, we propose an inf-sup stable method for the Stokes problem. Taking inspiration from the recent work of Galvin, Linke et al. [27, we additionally consider the problem of large irrotational body forces and pinpoint a strategy to preserve accuracy in the approximation of the velocity field for the case when a Helmholtz decomposition of volumetric body forces is available. We emphasize that this strategy is applicable to more general discretizations than the one considered in this work. Also in this case, suitable modifications of the right-hand side allow to establish a link with finite volumes, and nonconforming finite element methods on matching simplicial meshes.

The paper is organized as follows. In Section 2 we introduce the notation and define the concepts of admissible mesh sequence and pyramidal submesh inspired by [16,17. The main novelty of this section is the proof that the pyramidal submesh inherits the shape- and contact-regularity properties of the original mesh. Section 3 details the construction of the space $\mathfrak{C} \mathfrak{R}\left(\mathcal{K}_{h}\right)$ as well as the proofs of the properties (CR1) and (CR2). In Section 4 we present two applications of the space $\mathfrak{C} \Re\left(\mathcal{K}_{h}\right)$, namely the approximation of the linear elasticity problem for quasi-incompressible materials and the discretization of the Stokes equations with large irrotational body forces. Finally, in Appendix $\mathrm{A}$ we adapt the ideas of Section 3 to construct a discrete space which mimics the properties of the lowest-order Raviart-Thomas space on general polygonal or polyhedral meshes.

\section{Admissible mesh SeQUenCes}

Following [17, Chapter 1] and [16, Section 1], we introduce in this section the concept of admissible mesh sequence of a bounded connected polygonal or polyhedral domain $\Omega \subset \mathbb{R}^{d}, d \geqslant 2$. For the sake of brevity, we only give the proofs of the new results, and refer to [16,17] for further details.

2.1. Shape- and contact-regularity. Let $\mathcal{H} \subset \mathbb{R}_{*}^{+}$denote a countable set having 0 as its unique accumulation point. We consider mesh sequences $\mathcal{K}_{\mathcal{H}}:=\left(\mathcal{K}_{h}\right)_{h \in \mathcal{H}}$ where, for all $h \in \mathcal{H}, \mathcal{K}_{h}$ denotes a finite collection of nonempty disjoint open polyhedra $\mathcal{K}_{h}=\{K\}$ such that $\bar{\Omega}=\bigcup_{K \in \mathcal{K}_{h}} \bar{K}$ and $h=\max _{K \in \mathcal{K}_{h}} h_{K}\left(h_{K}\right.$ denotes here the diameter of the element $K \in \mathcal{K}_{h}$ ). We say that a hyperplanar closed connected subset $F$ of $\bar{\Omega}$ is a mesh face if it has positive $(d-1)$-dimensional measure and if either there exist $K_{1}, K_{2} \in \mathcal{K}_{h}$ such that $F \subset \partial K_{1} \cap \partial K_{2}$ (and $F$ is called an interface) or there exists $K \in \mathcal{K}_{h}$ such that $F \subset \partial K \cap \partial \Omega$ (and $F$ is called a boundary face). Interfaces are collected in the set $\mathcal{F}_{\mathcal{K}_{h}}^{\mathrm{i}}$, boundary faces in $\mathcal{F}_{\mathcal{K}_{h}}^{\mathrm{b}}$ and we let $\mathcal{F}_{\mathcal{K}_{h}}:=\mathcal{F}_{\mathcal{K}_{h}}^{\mathrm{i}} \cup \mathcal{F}_{\mathcal{K}_{h}}^{\mathrm{b}}$. The diameter of a face $F \in \mathcal{F}_{\mathcal{K}_{h}}$, is denoted by $h_{F}$. Moreover, we set, for all $K \in \mathcal{K}_{h}, \mathcal{F}_{K}:=\left\{F \in \mathcal{F}_{\mathcal{K}_{h}} \mid F \subset \partial K\right\}$. According to the context, the notation $|\cdot|$ is used for the $d$ - or the $(d-1)$-dimensional Lebesgue measure. In the rest of this section, we discuss some fairly general regularity conditions on the mesh sequence $\mathcal{K}_{\mathcal{H}}$ that allow us to prove basic results such as trace and inverse inequalities and polynomial approximation properties. 


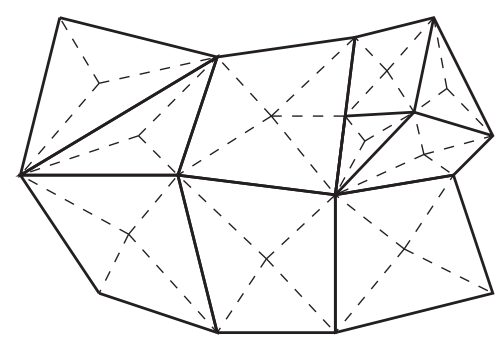

Figure 1. Mesh $\mathcal{K}_{h}$ (solid lines) and pyramidal submesh $\mathcal{P}_{h}$ (dashed lines)

Definition 1 (Shape- and contact-regularity). The mesh sequence $\mathcal{K}_{\mathcal{H}}$ is shapeand contact-regular if for all $h \in \mathcal{H}, \mathcal{K}_{h}$ admits a matching simplicial submesh $\mathcal{T}_{h}$ such that

(i) Shape-regularity. There exists a real $\varrho_{1}>0$ independent of $h$ such that, for all $h \in \mathcal{H}$ and all simplex $T \in \mathcal{T}_{h}$ of diameter $h_{T}$ and inradius $r_{T}$, there holds $\varrho_{1} h_{T} \leqslant r_{T}$.

(ii) Contact-regularity. There exists a real $\varrho_{2}>0$ independent of $h$ such that, for all $h \in \mathcal{H}$, all $K \in \mathcal{K}_{h}$, and all $T \in \mathcal{T}_{K}:=\left\{T \in \mathcal{T}_{h} \mid T \subset K\right\}$, there holds $\varrho_{2} h_{K} \leqslant h_{T}$.

2.2. Admissible mesh sequences. The discrete space introduced in this work requires to identify a set of points which play a pivotal role in the construction.

Definition 2 (Cell centers). The mesh sequence $\mathcal{K}_{\mathcal{H}}$ admits a set of cell centers if, for all $h \in \mathcal{H}$ and all $K \in \mathcal{K}_{h}$, there exists a point $\boldsymbol{x}_{K}$ such that $K$ is star-shaped with respect to $\boldsymbol{x}_{K}$ (the cell center) and, for all $F \in \mathcal{F}_{K}$, there holds,

$$
d_{K, F} \geqslant \varrho_{3} h_{K},
$$

where $d_{K, F}$ denotes the orthogonal distance between $\boldsymbol{x}_{K}$ and $F$ and $\varrho_{3}>0$ is independent of $h$.

Let $\mathcal{K}_{\mathcal{H}}$ admit a set of cell centers. We define for all $h \in \mathcal{H}$ the pyramidal submesh

$$
\mathcal{P}_{h}=\left\{K_{F}\right\}_{K \in \mathcal{K}_{h}, F \in \mathcal{F}_{K}},
$$

where, for all $K \in \mathcal{K}_{h}$ and all $F \in \mathcal{F}_{K}, K_{F}$ denotes the open pyramid of apex $\boldsymbol{x}_{K}$ and base $F$. An example of mesh $\mathcal{K}_{h}$ and associated pyramidal submesh $\mathcal{P}_{h}$ is provided in Figure 11 Each element of $\mathcal{P}_{h}$ is associated to a unique element $K \in \mathcal{K}_{h}$ and a unique face $F \in \mathcal{F}_{K}$. When this link is irrelevant, the generic element of $\mathcal{P}_{h}$ is noted $P$ instead of $K_{F}$. The pyramids $\left\{K_{F}\right\}_{K \in \mathcal{K}_{h}, F \in \mathcal{F}_{K}}$ are nondegenerate owing to assumption (11). In the two-dimensional case, $\mathcal{P}_{h}$ is matching and simplicial while, in higher dimension, it is in general not simplicial. Owing to the planarity of faces, there holds for all $K \in \mathcal{K}_{h}$ and all $F \in \mathcal{F}_{K}$,

$$
\left|K_{F}\right|=\frac{|F| d_{K, F}}{d}
$$

The set of faces of $\mathcal{P}_{h}$ (including the mesh faces in $\mathcal{F}_{\mathcal{K}_{h}}$ as well as the lateral faces of the pyramids) is denoted by $\mathcal{F}_{\mathcal{P}_{h}}$ and we let $\mathcal{F}_{\mathcal{P}_{h}}^{\mathrm{i}}:=\mathcal{F}_{\mathcal{P}_{h}} \backslash \mathcal{F}_{\mathcal{K}_{h}}^{\mathrm{b}}$ and $\mathcal{F}_{\mathcal{P}_{h}}^{\mathrm{b}}:=\mathcal{F}_{\mathcal{K}_{h}}^{\mathrm{b}}$. Additionally, for all $P \in \mathcal{P}_{h}$, we introduce the set $\mathcal{F}_{P}:=\left\{F \in \mathcal{F}_{\mathcal{P}_{h}} \mid F \subset \partial P\right\}$. 
Lemma 3 (Shape- and contact-regularity of the pyramidal submesh). Let $\mathcal{K}_{\mathcal{H}}$ admit a set of cell centers. Then, if $\mathcal{K}_{\mathcal{H}}$ is shape- and contact-regular, the same holds for $\mathcal{P}_{\mathcal{H}}$.

Proof. Let $h \in \mathcal{H}$. By assumption, $\mathcal{K}_{h}$ admits a matching simplicial submesh $\mathcal{T}_{h}$. A matching simplicial submesh $\mathfrak{T}_{h}$ of the pyramidal submesh $\mathcal{P}_{h}$ can be constructed as follows: For all $K \in \mathcal{K}_{h}$ and all $F \in \mathcal{F}_{K}$ (i) a $(d-1)$-simplicial mesh $\mathfrak{S}_{F}$ of $F$ is obtained taking the trace of $\mathcal{T}_{h}$ on $F$; (ii) a $d$-simplicial mesh $\mathfrak{T}_{K_{F}}$ of the pyramid $K_{F}$ is then obtained connecting the (hyperplanar) elements in $\mathfrak{S}_{F}$ to the cell center. A matching simplicial submesh of $\mathcal{P}_{h}$ is obtained by setting

$$
\mathfrak{T}_{h}:=\bigcup_{K \in \mathcal{K}_{h}, F \in \mathcal{F}_{K}} \mathfrak{T}_{K_{F}} .
$$

(i) Shape-regularity. We prove that there exists a real $\varrho_{1}^{\prime}>0$ independent of $h$ such that $\varrho_{1}^{\prime} h_{T} \leqslant r_{T}$ for all $T \in \mathfrak{T}_{h}$. Let $K_{F} \in \mathcal{P}_{h}$ and $T \in \mathfrak{T}_{K_{F}}$ be given. Denoting by $r_{T}$ the inradius of $T$, letting $\mathcal{A}_{T}:=|\partial T|$ and $\sigma:=\partial T \cap F$, there holds $d|T|=r_{T} \mathcal{A}_{T}=|\sigma| d_{K, F}$, hence

$$
r_{T}=\frac{|\sigma| d_{K, F}}{\mathcal{A}_{T}}
$$

Since the $(d-1)$-dimensional measure of each face of $T$ is bounded by $h_{K}^{d-1}$ and $T$ has $(d+1)$ faces, there holds $\mathcal{A}_{T} \leqslant(d+1) h_{K}^{d-1}$. Now let $S \in \mathcal{T}_{h}$ be the unique simplex such that $\partial S \cap F=\sigma$ and $S \subset K$. Denoting by $r_{\sigma}$ the inradius of $\sigma$, and observing that $r_{\sigma} \geqslant r_{S}$ by a simple argument based on the Pythagorean theorem, it is inferred $|\sigma| \geqslant\left|\mathfrak{B}_{d-1}\right| r_{\sigma}^{d-1} \geqslant\left|\mathfrak{B}_{d-1}\right| r_{S}^{d-1} \geqslant\left|\mathfrak{B}_{d-1}\right|\left(\varrho_{1} \varrho_{2}\right)^{d-1} h_{K}^{d-1}$ owing to the shape- and contact-regularity of $\mathcal{K}_{h}\left(\mathfrak{B}_{d-1}\right.$ denotes here the $(d-1)$-dimensional unit ball). Plugging these inequalities into (3), it is inferred

$$
r_{T} \geqslant \frac{\left|\mathfrak{B}_{d-1}\right|\left(\varrho_{1} \varrho_{2}\right)^{d-1}}{d+1} d_{K, F} \geqslant \varrho_{3} \frac{\left|\mathfrak{B}_{d-1}\right|\left(\varrho_{1} \varrho_{2}\right)^{d-1}}{d+1} h_{T}
$$

and the conclusion follows with $\varrho_{1}^{\prime}=\varrho_{3}\left|\mathfrak{B}_{d-1}\right|\left(\varrho_{1} \varrho_{2}\right)^{d-1} /(d+1)$.

(ii) Contact-regularity. We prove that there exists a real $\varrho_{2}^{\prime}>0$ independent of $h$ such that, for all $K_{F} \in \mathcal{P}_{h}$ and all $T \in \mathfrak{T}_{K_{F}}, \varrho_{2}^{\prime} h_{K_{F}} \leqslant h_{T}$. To this end, we invoke (11) to infer, for all $K_{F} \in \mathcal{P}_{h}$ and all $T \in \mathfrak{T}_{K_{F}}, h_{T} \geqslant d_{K, F} \geqslant \varrho_{3} h_{K} \geqslant \varrho_{3} h_{K_{F}}$, where $h_{K_{F}}$ denotes the diameter of $K_{F}$. The conclusion follows with $\varrho_{2}^{\prime}=\varrho_{3}$.

We close this section with the following definition.

Definition 4 (Admissible mesh sequence). The mesh sequence $\mathcal{K}_{\mathcal{H}}$ is admissible if it is shape- and contact-regular and it admits a set of cell centers. For an admissible mesh sequence, the reals $\varrho_{1}, \varrho_{2}$, and $\varrho_{3}$, are collectively referred to as mesh regularity parameters.

2.3. Broken function spaces and polynomial approximation. For $\mathcal{S}_{h} \in$ $\left\{\mathcal{K}_{h}, \mathcal{P}_{h}\right\}$ and an integer $k \geqslant 0$, we introduce the broken polynomial space

$$
\mathbb{P}_{d}^{k}\left(\mathcal{S}_{h}\right):=\left\{v \in L^{2}(\Omega) \mid \forall S \in \mathcal{S}_{h}, v_{\mid S} \in \mathbb{P}_{d}^{k}(S)\right\},
$$

where $\mathbb{P}_{d}^{k}$ denotes the space of polynomial functions of total degree at most $k$. Broken polynomial spaces are a special instance of broken Sobolev spaces: For an integer $l \geqslant 1$,

$$
H^{l}\left(\mathcal{S}_{h}\right):=\left\{v \in L^{2}(\Omega) \mid \forall S \in \mathcal{S}_{h}, v_{\mid S} \in H^{l}(S)\right\} .
$$


We define the broken gradient denoted by $\nabla_{h}$ and acting on functions $v \in H^{1}\left(\mathcal{S}_{h}\right)$ such that $\left(\nabla_{h} v\right)_{\mid S}:=\nabla\left(v_{\mid S}\right)$, for all $S \in \mathcal{S}_{h}$. We also define the broken divergence of a vector-valued field $\boldsymbol{v} \in H^{1}\left(\mathcal{S}_{h}\right)^{d}$ denoted by $\nabla_{h} \cdot \boldsymbol{v}$, and the broken symmetric gradient $\underline{\underline{\varepsilon_{h}}}(\boldsymbol{v})$, respectively, as the trace and as the symmetric part of the broken tensor-gradient $\boldsymbol{\nabla}_{h} \boldsymbol{v}$. The shape- and contact-regularity of the mesh sequences $\mathcal{K}_{\mathcal{H}}$ and $\mathcal{P}_{\mathcal{H}}$ are instrumental to prove the following result; see [17, Lemmata 1.46 and 1.49].

Lemma 5 (Trace inequalities). Let $\mathcal{K}_{\mathcal{H}}$ be an admissible mesh sequence, and denote by $\mathcal{P}_{\mathcal{H}}$ the corresponding sequence of pyramidal submeshes. Then, there exist two reals $C_{\mathrm{tr}}$ and $C_{\mathrm{tr}, \mathrm{c}}$ independent of $h$ such that, for all $h \in \mathcal{H}$ with $\mathcal{S}_{h} \in\left\{\mathcal{K}_{h}, \mathcal{P}_{h}\right\}$

$$
\forall v_{h} \in \mathbb{P}_{d}^{k}\left(\mathcal{P}_{h}\right), \forall P \in \mathcal{P}_{h}, \forall F \in \mathcal{F}_{P}, \quad\left\|v_{h}\right\|_{L^{2}(F)} \leqslant C_{\mathrm{tr}} h_{F}^{-1 / 2}\left\|v_{h}\right\|_{L^{2}(P)},
$$

$$
\forall v \in H^{1}\left(\mathcal{S}_{h}\right), \forall S \in \mathcal{S}_{h}, \forall F \in \mathcal{F}_{S}, \quad\|v\|_{L^{2}(F)} \leqslant C_{\mathrm{tr}, \mathrm{c}}\left(h_{S}^{-1}\|v\|_{L^{2}(S)}^{2}+h_{S}|v|_{H^{1}(S)}^{2}\right)^{1 / 2} .
$$

For every interface $F \in \mathcal{F}_{\mathcal{S}_{h}}^{\mathrm{i}}, \mathcal{S}_{h} \in\left\{\mathcal{K}_{h}, \mathcal{P}_{h}\right\}$, we introduce an arbitrary but fixed ordering of the elements $S_{1}$ and $S_{2}$ such that $F \subset \partial S_{1} \cap \partial S_{2}$ and let $\boldsymbol{n}_{F}:=\boldsymbol{n}_{S_{1}, F}=$ $-\boldsymbol{n}_{S_{2}, F}$, where $\boldsymbol{n}_{S_{i}, F}, i \in\{1,2\}$, denotes the unit normal to $F$ pointing out of $S_{i}$. The orientation of the normal remains coherent when $F \in \mathcal{F}_{\mathcal{K}_{h}}^{\mathrm{i}}$ is regarded as an element of $\mathcal{F}_{\mathcal{P}_{h}}^{\mathrm{i}}$. For all $S \in \mathcal{S}_{h}$, we also introduce the symbol $\boldsymbol{n}_{S}$ to denote the vector-valued field such that $\boldsymbol{n}_{S \mid F}=\boldsymbol{n}_{S, F}$ for all $F \in \mathcal{F}_{S}$. On boundary faces $F \in \mathcal{F}_{\mathcal{P}_{h}}^{\mathrm{b}}, \boldsymbol{n}_{F}$ denotes the unit normal pointing out of $\Omega$.

We next introduce jump and average trace operators that are widely used in the context of nonconforming finite element methods. For a face $F \in \mathcal{F}_{\mathcal{P}_{h}}^{\mathrm{i}}$ with $F \subset \partial P_{1} \cap \partial P_{2}$ and a scalar-valued function $v$ admitting a possibly two-valued trace on $F$ we set,

$$
\llbracket v \rrbracket_{F}:=v_{\mid P_{1}}-v_{\mid P_{2}}, \quad\{v\}_{F}:=\frac{1}{2}\left(v_{\mid P_{1}}+v_{\mid P_{2}}\right) .
$$

If $F \in \mathcal{F}_{\mathcal{P}_{h}}^{\mathrm{b}}$ with $F=\partial P \cap \partial \Omega$, we conventionally set $\llbracket v \rrbracket_{F}=\{v\}_{F}:=v_{\mid P}$. When applied to vector-valued functions, both the jump and average operators act componentwise. Whenever no confusion can arise, we omit the subscript $F$ and simply write $\llbracket v \rrbracket,\{v\}$.

We close this section by considering polynomial approximation on admissible mesh sequences. It has been proved in [17, Lemma 1.40] that, for a shape- and contact-regular mesh sequence, the number of simplices from the submesh $\mathcal{T}_{h}$ contained in each element $K \in \mathcal{K}_{h}$ is bounded uniformly in $h$. This, together with the results of Dupont and Scott [23, yields the following.

Lemma 6 (Optimal polynomial approximation). Let $\mathcal{K}_{\mathcal{H}}$ denote a shape- and contact-regular mesh sequence. Then, for all $h \in \mathcal{H}$, all $K \in \mathcal{K}_{h}$, all polynomial degree $k \geqslant 0$, all $s \in\{0, \ldots, k+1\}$ and all $v \in H^{s}(K)$, there holds with $\Pi_{h}^{k}$ denoting the $L^{2}$-orthogonal projector onto $\mathbb{P}_{d}^{k}\left(\mathcal{K}_{h}\right)$,

$$
\left|v-\Pi_{h}^{k} v\right|_{H^{m}(K)} \leqslant C_{\text {app }} h_{K}^{s-m}|v|_{H^{s}(K)} \quad \forall m \in\{0, \ldots, s\},
$$

where $C_{\text {app }}$ is independent of both $K$ and $h$. 
We also note the following result, which is an immediate consequence of the trace inequality (6) with $\mathcal{S}_{h}=\mathcal{K}_{h}$ and of the approximation properties of the $L^{2}$-orthogonal projector.

Proposition 7 (Approximation on mesh faces). For an admissible mesh sequence $\mathcal{K}_{\mathcal{H}}$ there holds for all $h \in \mathcal{H}$, all $K \in \mathcal{K}_{h}$, all $F \in \mathcal{F}_{K}$, all polynomial degree $k \geqslant 0$, all $s \in\{0, \ldots, k+1\}$, and all $v \in H^{s}(K)$,

$$
\left\|v-\Pi_{h}^{k} v\right\|_{L^{2}(F)} \leqslant C h_{K}^{s-1 / 2}|v|_{H^{s}(K)},
$$

where $C=C_{t r, c} C_{\text {app }}$ with $C_{t r, c}$ defined as in (6) and $C_{\text {app }}$ as in (17).

\section{Mimicking the properties of the lowest-order CrouzeiX-Raviart SPACE ON GENERAL MESHES}

In this section we present a piecewise affine functional space obtained in the spirit of [16] that extends the properties (CR1) and (CR2) of the Crouzeix-Raviart space [15] to general polygonal or polyhedral meshes.

3.1. Construction. In the spirit of ccG methods, we proceed in three steps by prescribing: (i) the vector space of degrees of freedom $\mathbb{V}_{h}$; (ii) a gradient reconstruction $\mathfrak{G}_{h}$, piecewise constant on $\mathcal{P}_{h}$; (iii) a piecewise affine reconstruction $\mathfrak{R}_{h}$ on $\mathcal{P}_{h}$, based on the gradient $\mathfrak{G}_{h}$. As for HFV methods [25], the vector space of DOFs contains cell and face unknowns and is defined by

$$
\mathbb{V}_{h}:=\left\{\nabla_{h}=\left(\left(v_{K} \in \mathbb{R}\right)_{K \in \mathcal{K}_{h}},\left(v_{F} \in \mathbb{R}\right)_{F \in \mathcal{F}_{\mathcal{K}_{h}}}\right) \in \mathbb{R}^{\mathcal{K}_{h}} \times \mathbb{R}^{\mathcal{F}_{\mathcal{K}_{h}}}\right\} .
$$

The gradient operator generalizes the one of [25], and is composed of a consistent contribution piecewise constant on the primal mesh $\mathcal{K}_{h}$ plus a subgrid correction piecewise constant on the pyramidal submesh $\mathcal{P}_{h}$. More precisely, $\mathfrak{G}_{h}: \mathbb{V}_{h} \rightarrow$ $\mathbb{P}_{d}^{0}\left(\mathcal{P}_{h}\right)^{d}$ realizes the mapping $\nabla_{h} \mapsto \mathfrak{G}_{h}\left(\nabla_{h}\right)$ with

$$
\mathfrak{G}_{h}\left(\nabla_{h}\right)_{\mid K_{F}}=\boldsymbol{G}_{K_{F}}\left(\mathbb{v}_{h}\right):=\boldsymbol{G}_{K}\left(\nabla_{h}\right)+\boldsymbol{R}_{K_{F}}\left(\nabla_{h}\right), \quad \forall K \in \mathcal{K}_{h}, F \in \mathcal{F}_{K},
$$

where, letting $\overline{\boldsymbol{x}}_{F}:=\langle\boldsymbol{x}\rangle_{F}$ (for a function $\varphi$ integrable on $F$, we define $\langle\varphi\rangle_{F}:=$ $\left.\int_{F} \varphi /|F|\right)$,

$$
\begin{gathered}
\boldsymbol{G}_{K}\left(\nabla_{h}\right):=\frac{1}{|K|} \sum_{F \in \mathcal{F}_{K}}|F| v_{F} \boldsymbol{n}_{K, F}, \\
\boldsymbol{R}_{K_{F}}\left(\nabla_{h}\right):=\frac{\eta}{d_{K, F}}\left(v_{F}-v_{K}-\boldsymbol{G}_{K}\left(\nabla_{h}\right) \cdot\left(\overline{\boldsymbol{x}}_{F}-\boldsymbol{x}_{K}\right)\right) \boldsymbol{n}_{K, F},
\end{gathered}
$$

and $\eta>0$ is a user-dependent parameter. With a slight abuse in notation, the symbols $\boldsymbol{G}_{K_{F}}\left(\nabla_{h}\right), \boldsymbol{G}_{K}\left(\nabla_{h}\right)$, and $\boldsymbol{R}_{K_{F}}\left(\nabla_{h}\right)$ will also be used to denote the corresponding constant fields on $K_{F}, K$, and $K_{F}$, respectively. The reconstruction operator $\mathfrak{R}_{h}: \mathbb{V}_{h} \rightarrow \mathbb{P}_{d}^{1}\left(\mathcal{P}_{h}\right)$ realizes the mapping $\nabla_{h} \mapsto \mathfrak{R}_{h}\left(\mathbb{\vee}_{h}\right)$ with

$$
\mathfrak{R}_{h}\left(\nabla_{h}\right)_{\mid K_{F}}(\boldsymbol{x})=v_{F}+\mathfrak{G}_{h}\left(\nabla_{h}\right)_{\mid K_{F}} \cdot\left(\boldsymbol{x}-\overline{\boldsymbol{x}}_{F}\right), \quad \forall K_{F} \in \mathcal{P}_{h}, \forall \boldsymbol{x} \in K_{F} .
$$

By construction, there holds $\nabla_{h} \Re_{h}=\mathfrak{G}_{h}$. We emphasize that, in view of Lemma 8 below, the affine reconstruction in $K_{F}$ is obtained by perturbing the face unknown $v_{F}$, unlike [16], where the cell unknown $v_{K}$ is used instead. We are now ready to introduce the discrete space

$$
\mathfrak{C} \mathfrak{R}\left(\mathcal{K}_{h}\right):=\mathfrak{R}_{h}\left(\mathbb{V}_{h}\right)
$$




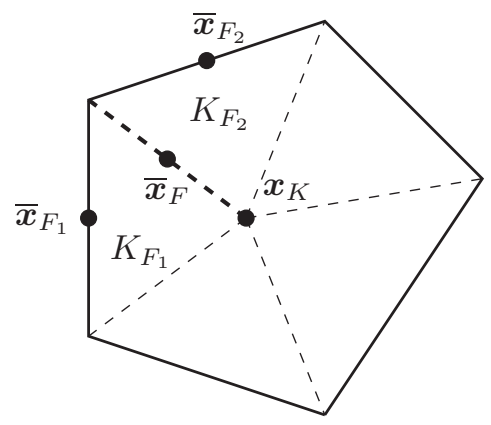

(A) Element $K$ (solid line), elementary pyramidal submesh (dashed line), and lateral pyramidal face $F$ shared by the pyramids $K_{F_{1}}$ and $K_{F_{2}}$ (thick dashed line)

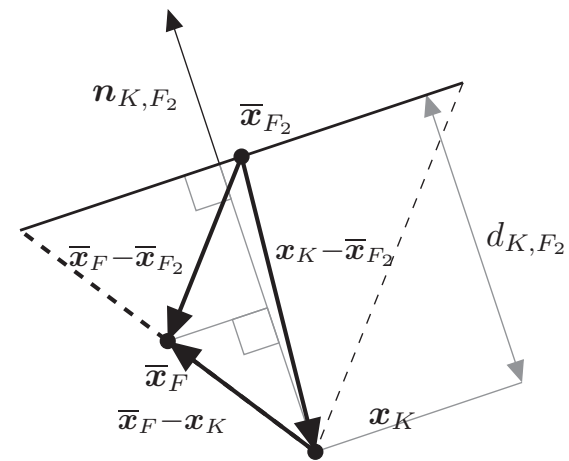

(B) Zoom on the pyramid $K_{F_{2}}$

Figure 2. Notation for the proof of Lemma 8

3.2. Continuity of mean values at interfaces. In this section we prove that the choice $\eta=d$ in (9) yields the continuity of the mean values (or, equivalently, the barycentric values) of discrete functions across all the interfaces in $\mathcal{F}_{\mathcal{P}_{h}}^{\mathrm{i}}$ (including lateral pyramidal faces). This is in contrast with the choice $\eta=d^{1 / 2}$ advocated in 25] to recover the two-point finite volume scheme on superadmissible meshes.

Lemma 8 (Continuity of mean values at interfaces). Let $\mathcal{K}_{h}$ belong to an admissible mesh sequence and, if $\mathcal{K}_{h}$ is not matching simplicial, assume $\eta=d$ in (9). Then, there holds for all $v_{h} \in \mathfrak{C} \mathfrak{R}\left(\mathcal{K}_{h}\right)$,

$$
\forall F \in \mathcal{F}_{\mathcal{P}_{h}}^{\mathrm{i}}, \quad\left\langle\llbracket v_{h} \rrbracket\right\rangle_{F}=0 .
$$

Proof. Let $F \in \mathcal{F}_{\mathcal{P}_{h}}^{\mathrm{i}}, \nabla_{h} \in \mathbb{V}_{h}$, and set $v_{h}:=\mathfrak{R}_{h}\left(\nabla_{h}\right) \in \mathfrak{C} \mathfrak{R}\left(\mathcal{K}_{h}\right)$. We distinguish two cases.

(i) If $F \in \mathcal{F}_{\mathcal{K}_{h}}^{\mathrm{i}}$ is a face of the primal mesh $\mathcal{K}_{h}$, the fact that $\left\langle\llbracket v_{h} \rrbracket\right\rangle_{F}=0$ is an immediate consequence of choosing $v_{F}$ as a starting point in (10).

(ii) If $F \in \mathcal{F}_{\mathcal{P}_{h}}^{\mathrm{i}} \backslash \mathcal{F}_{\mathcal{K}_{h}}^{\mathrm{i}}$ is a lateral pyramidal face, there exist a unique element $K \in \mathcal{K}_{h}$ and two faces $F_{1}, F_{2} \in \mathcal{F}_{K}$ such that $F \subset \partial K_{F_{1}} \cap \partial K_{F_{2}}$ (cf. Figure 2a).

There holds for $i \in\{1,2\}$ (cf. Figure 2b),

$$
\begin{aligned}
\left(\overline{\boldsymbol{x}}_{F}-\overline{\boldsymbol{x}}_{F_{i}}\right) \cdot \boldsymbol{n}_{K, F_{i}} & =\left(\overline{\boldsymbol{x}}_{F}-\boldsymbol{x}_{K}\right) \cdot \boldsymbol{n}_{K, F_{i}}+\left(\boldsymbol{x}_{K}-\overline{\boldsymbol{x}}_{F_{i}}\right) \cdot \boldsymbol{n}_{K, F_{i}} \\
& =\left(\frac{d-1}{d}-1\right) d_{K, F_{i}}=-\frac{d_{K, F_{i}}}{d}
\end{aligned}
$$

where we have used the fact that $\overline{\boldsymbol{x}}_{F}$ is the barycenter of the $(d-1)$-simplex $F$ to treat the term $\left(\overline{\boldsymbol{x}}_{F}-\boldsymbol{x}_{K}\right) \cdot \boldsymbol{n}_{K, F_{i}}$. Using the above result together with (9)) it is inferred for $i \in\{1,2\}$,

$$
\alpha_{i}:=\boldsymbol{R}_{K_{F_{i}}}\left(\nabla_{h}\right) \cdot\left(\overline{\boldsymbol{x}}_{F}-\overline{\boldsymbol{x}}_{F_{i}}\right)=-\frac{\eta}{d}\left(v_{F_{i}}-v_{K}-\boldsymbol{G}_{K}\left(\nabla_{h}\right) \cdot\left(\overline{\boldsymbol{x}}_{F_{i}}-\boldsymbol{x}_{K}\right)\right) .
$$


Using the definition of the jump operator and substituting the expression (10) for the barycentric values $v_{h \mid K_{F_{i}}}\left(\overline{\boldsymbol{x}}_{F}\right), i \in\{1,2\}$, we obtain

$$
\begin{aligned}
\left\langle\llbracket v_{h} \rrbracket\right\rangle_{F} & =v_{h \mid K_{F_{1}}}\left(\overline{\boldsymbol{x}}_{F}\right)-v_{h \mid K_{F_{2}}}\left(\overline{\boldsymbol{x}}_{F}\right) \\
& =v_{F_{1}}-v_{F_{2}}-\boldsymbol{G}_{K}\left(\nabla_{h}\right) \cdot\left(\overline{\boldsymbol{x}}_{F_{1}}-\overline{\boldsymbol{x}}_{F_{2}}\right)+\alpha_{1}-\alpha_{2} \\
& =\left(1-\frac{\eta}{d}\right)\left(v_{F_{1}}-v_{F_{2}}-\boldsymbol{G}_{K}\left(\nabla_{h}\right) \cdot\left(\overline{\boldsymbol{x}}_{F_{1}}-\overline{\boldsymbol{x}}_{F_{2}}\right)\right) .
\end{aligned}
$$

If $\mathcal{K}_{h}$ is a matching simplicial mesh, $\boldsymbol{G}_{K}\left(\nabla_{h}\right)$ coincides by definition with the standard Crouzeix-Raviart gradient (cf. also Proposition 9), hence the second factor in (12) vanishes, otherwise the assumption $\eta=d$ is needed to infer $\left\langle\llbracket v_{h} \rrbracket\right\rangle_{F}=0$, thereby concluding the proof.

The following result establishes a link with the classical Crouzeix-Raviart space $\mathbb{C R}\left(\mathcal{K}_{h}\right)$.

Proposition 9 (The matching simplicial case). Assume that $\mathcal{K}_{h}$ is a matching simplicial mesh. Then, for all $\eta>0$ in (9) there holds

$$
\mathbb{C R}\left(\mathcal{K}_{h}\right) \subset \mathfrak{C} \mathfrak{R}\left(\mathcal{K}_{h}\right)
$$

Proof. Let $v_{h} \in \mathbb{C} \mathbb{R}\left(\mathcal{K}_{h}\right)$ and set $\nabla_{h}:=\left(\left(v_{h}\left(\boldsymbol{x}_{K}\right)\right)_{K \in \mathcal{K}_{h}},\left(v_{h}\left(\overline{\boldsymbol{x}}_{F}\right)\right)_{F \in \mathcal{F}_{\mathcal{K}_{h}}}\right)$. By definition there holds (cf. (9) ) $\boldsymbol{G}_{K}\left(\nabla_{h}\right)=\left(\nabla_{h} v_{h}\right)_{\mid K}$ for all $K \in \mathcal{K}_{h}$. Using the linearity of $v_{h}$ inside each element it is inferred $\boldsymbol{R}_{K_{F}}\left(\nabla_{h}\right)=\mathbf{0}$, hence $\boldsymbol{G}_{K_{F}}\left(\nabla_{h}\right)=\boldsymbol{G}_{K}\left(\nabla_{h}\right)=$ $\left(\nabla_{h} v_{h}\right)_{\mid K}$ for all $F \in \mathcal{F}_{K}$. As a consequence, we conclude that $v_{h}=\mathfrak{R}_{h}\left(\nabla_{h}\right) \in$ $\mathfrak{C} \mathfrak{R}\left(\mathcal{K}_{h}\right)$.

3.3. Approximation. In this section we introduce a suitable interpolator on $\mathfrak{C} \Re\left(\mathcal{K}_{h}\right)$ and study its approximation properties. Let $\mathcal{I}_{h}^{\mathfrak{C} \Re}: H^{1}(\Omega) \rightarrow \mathfrak{C} \Re\left(\mathcal{K}_{h}\right)$ be such that, for all $v \in H^{1}(\Omega), \mathcal{I}_{h}^{\mathfrak{C} R}(v)=\mathfrak{R}_{h}\left(\nabla_{h}\right)$ with

$$
\mathbb{V}_{h} \ni \nabla_{h}=\left(\left(\Pi_{h}^{1} v\left(\boldsymbol{x}_{K}\right)\right)_{K \in \mathcal{K}_{h}},\left(\langle v\rangle_{F}\right)_{F \in \mathcal{F}_{\mathcal{K}_{h}}}\right) .
$$

When applied to vector-valued fields, $\mathcal{I}_{h}^{\mathfrak{C} \mathfrak{R}}$ acts componentwise.

Lemma 10 (Approximation in $\left.\mathfrak{C} \mathfrak{R}\left(\mathcal{K}_{h}\right)\right)$. For all $\eta>0$ in (9) and all $v \in H^{1}(\Omega)$ there holds with $v_{h}:=\mathcal{I}_{h}^{\mathfrak{C} \Re}(v) \in \mathfrak{C} \Re\left(\mathcal{K}_{h}\right)$,

$$
\Pi_{h}^{0}\left(\nabla_{h} v_{h}\right)=\Pi_{h}^{0}(\boldsymbol{\nabla} v)
$$

where $\Pi_{h}^{0}$ denotes the $L^{2}$-orthogonal projector on $\mathbb{P}_{d}^{0}\left(\mathcal{K}_{h}\right)^{d}$. Moreover, there exists a real $C>0$ independent of the meshsize such that, for all $h \in \mathcal{H}$, all $K \in \mathcal{K}_{h}$, all $v \in H^{1}(\Omega) \cap H^{l+1}\left(\mathcal{K}_{h}\right), l \in\{0,1\}$, there holds, with $v_{h}:=\mathcal{I}_{h}^{\mathfrak{C} \Re}(v)$,

$$
\left\|v-v_{h}\right\|_{L^{2}(K)}+h_{K}\left\|\boldsymbol{\nabla} v-\nabla_{h} v_{h}\right\|_{L^{2}(K)^{d}} \leqslant C h_{K}^{l+1}|v|_{H^{l+1}(K)} .
$$

Proof. To avoid naming generic constants, we use the notation $a \lesssim b$ for the inequality $a \leqslant C b$ with $C>0$ independent of the meshsize.

(i) Equality (15). For a given $v \in H^{1}(\Omega)$, let $\nabla_{h}$ be defined as in (14). We start by noting the following orthogonality relation (cf. [25, eq. (27)]) valid for all $\mathfrak{w}_{h} \in \mathbb{V}_{h}$ and all $K \in \mathcal{K}_{h}$ :

$$
\sum_{F \in \mathcal{F}_{K}}\left|K_{F}\right| \boldsymbol{R}_{K_{F}}\left(\mathbb{W}_{h}\right)=\mathbf{0}
$$


As a consequence, for all $K \in \mathcal{K}_{h}$ there holds,

$$
\Pi_{h}^{0}\left(\boldsymbol{\nabla}_{h} v_{h}\right)_{\mid K}=\boldsymbol{G}_{K}\left(\nabla_{h}\right)=\frac{1}{|K|} \sum_{F \in \mathcal{F}_{K}}|F|\langle v\rangle_{F} \boldsymbol{n}_{K, F}=\frac{1}{|K|} \int_{\partial K} v \boldsymbol{n}_{K}=\Pi_{h}^{0}(\boldsymbol{\nabla} v)_{\mid K},
$$

where we have used the planarity of faces and Green's formula. Relation (15) follows.

(ii) Inequality (16). Let $v \in H^{1}(\Omega) \cap H^{l+1}\left(\mathcal{K}_{h}\right)$ and define $\nabla_{h}$ as in (14). We first estimate $\left\|\nabla v-\nabla_{h} v_{h}\right\|_{L^{2}(K)^{d}}, K \in \mathcal{K}_{h}$. Using (8), the previous point, and the triangular inequality we infer

$$
\begin{aligned}
\left\|\boldsymbol{\nabla} v-\nabla_{h} v_{h}\right\|_{L^{2}(K)^{d}} \leqslant & \left\|\nabla v-\Pi_{h}^{0}(\boldsymbol{\nabla} v)\right\|_{L^{2}(K)^{d}} \\
& +\left(\sum_{F \in \mathcal{F}_{K}}\left|K_{F}\right|\left|\boldsymbol{R}_{K_{F}}\left(\nabla_{h}\right)\right|^{2}\right)^{\frac{1}{2}}:=\mathfrak{T}_{1}+\mathfrak{T}_{2} .
\end{aligned}
$$

Using the approximation properties of the $L^{2}$-orthogonal projector it is readily inferred that $\mathfrak{T}_{1} \lesssim h_{K}^{l}|v|_{H^{l+1}(K)}$. To estimate the second term, we preliminarily observe that there holds for all $F \in \mathcal{F}_{K}$ with $w_{h}:=\Pi_{h}^{1} v\left(\Pi_{h}^{1}\right.$ denotes here the $L^{2}$-orthogonal projector on $\left.\mathbb{P}_{d}^{1}\left(\mathcal{K}_{h}\right)\right)$,

$$
\begin{aligned}
\boldsymbol{R}_{K_{F}}\left(\nabla_{h}\right) & =\frac{\eta}{d_{K, F}}\left(\langle v\rangle_{F}-w_{h}\left(\boldsymbol{x}_{K}\right)-\boldsymbol{G}_{K}\left(\nabla_{h}\right) \cdot\left(\overline{\boldsymbol{x}}_{F}-\boldsymbol{x}_{K}\right)\right) \boldsymbol{n}_{K, F} \\
& =\frac{\eta}{d_{K, F}}\left(\alpha_{K, F}+\beta_{K, F}\right) \boldsymbol{n}_{K, F},
\end{aligned}
$$

where $\alpha_{K, F}:=\langle v\rangle_{F}-\left\langle w_{h \mid K}\right\rangle_{F}, \beta_{K, F}:=\left(\nabla w_{h \mid K}-\boldsymbol{G}_{K}\left(\nabla_{h}\right)\right) \cdot\left(\overline{\boldsymbol{x}}_{F}-\boldsymbol{x}_{K}\right)$, and, since $w_{h \mid K}$ is affine in $K, w_{h}\left(\boldsymbol{x}_{K}\right)=\left\langle w_{h \mid K}\right\rangle_{F}-\boldsymbol{\nabla} w_{h \mid K} \cdot\left(\overline{\boldsymbol{x}}_{F}-\boldsymbol{x}_{K}\right)$. There follows from equation (18),

$$
\mathfrak{T}_{2}^{2} \lesssim \sum_{F \in \mathcal{F}_{K}} \frac{\left|K_{F}\right|}{d_{K, F}^{2}}\left|\alpha_{K, F}\right|^{2}+\sum_{F \in \mathcal{F}_{K}} \frac{\left|K_{F}\right|}{d_{K, F}^{2}}\left|\beta_{K, F}\right|^{2}:=\mathfrak{T}_{2,1}+\mathfrak{T}_{2,2} .
$$

Using (21), the Cauchy-Schwarz inequality, the mesh regularity assumption (11), the fact that $\operatorname{card}\left(\mathcal{F}_{K}\right)$ is bounded uniformly in $h$ (cf. [17, Lemma 1.41]), and Proposition 1 it is inferred,

$$
\begin{aligned}
\mathfrak{T}_{2,1} & =\frac{1}{d} \sum_{F \in \mathcal{F}_{K}} \frac{1}{d_{K, F}|F|}\left(\int_{F} v-w_{h}\right)^{2} \\
& \leqslant \frac{1}{d \varrho_{3}} \sum_{F \in \mathcal{F}_{K}} \frac{1}{h_{K}}\left\|v-w_{h}\right\|_{L^{2}(F)}^{2} \lesssim h_{K}^{2 l}|v|_{H^{l+1}(K)}^{2} .
\end{aligned}
$$

On the other hand, since $\left|\overline{\boldsymbol{x}}_{F}-\boldsymbol{x}_{K}\right| \leqslant h_{K}$ and both $\boldsymbol{\nabla} w_{h \mid K}$ and $\boldsymbol{G}_{K}\left(\mathbb{v}_{h}\right)$ are constant on $K$, there holds

$$
\begin{aligned}
\mathfrak{T}_{2,2} & \leqslant \sum_{F \in \mathcal{F}_{K}}\left|K_{F}\right| \frac{h_{K}^{2}}{d_{K, F}^{2}}\left|\nabla w_{h \mid K}-\boldsymbol{G}_{K}\left(\nabla_{h}\right)\right|^{2} \\
& \leqslant \frac{1}{\varrho_{3}^{2}}\left\|\nabla w_{h \mid K}-\Pi_{h}^{0}(\nabla v)\right\|_{L^{2}(K)^{d}}^{2} \lesssim h_{K}^{2 l}|v|_{H^{l+1}(K)}^{2},
\end{aligned}
$$


where we have used the mesh regularity assumption (10) together with (15), and concluded using the approximation properties of the $L^{2}$-orthogonal projector. Gathering up the bounds on $\mathfrak{T}_{1}$ and $\mathfrak{T}_{2}$ it is inferred

$$
\left\|\nabla v-\nabla_{h} v_{h}\right\|_{L^{2}(K)^{d}} \lesssim h_{K}^{l}|v|_{H^{l+1}(K)} .
$$

To complete the proof of inequality (16) it only remains to estimate $\left\|v-v_{h}\right\|_{L^{2}(K)}$. To this end, letting again $w_{h}:=\Pi_{h}^{1} v$, we apply the triangular inequality to infer

$$
\left\|v-v_{h}\right\|_{L^{2}(K)} \leqslant\left\|v-w_{h}\right\|_{L^{2}(K)}+\left\|w_{h}-v_{h}\right\|_{L^{2}(K)}:=\mathfrak{T}_{1}+\mathfrak{T}_{2} .
$$

The approximation properties of the $L^{2}$-orthogonal projector readily yield $\mathfrak{T}_{1} \lesssim$ $h_{K}^{l+1}|v|_{H^{l+1}(K)}$. For the second term, we notice that for all $F \in \mathcal{F}_{K}$ and all $\boldsymbol{x} \in K_{F}$, the linearity of both $w_{h \mid K}$ and $v_{h \mid K_{F}}$ yields

$$
w_{h \mid K}(\boldsymbol{x})=\left\langle w_{h \mid K}\right\rangle_{F}+\nabla w_{h \mid K} \cdot\left(\boldsymbol{x}-\overline{\boldsymbol{x}}_{F}\right), \quad v_{h \mid K_{F}}(\boldsymbol{x})=\langle v\rangle_{F}+\nabla v_{h \mid K_{F}} \cdot\left(\boldsymbol{x}-\overline{\boldsymbol{x}}_{F}\right) .
$$

As a consequence,

$$
\begin{aligned}
\left\|w_{h}-v_{h}\right\|_{L^{2}\left(K_{F}\right)}^{2} & \lesssim \int_{K_{F}}\left(\left\langle w_{h \mid K}-v\right\rangle_{F}\right)^{2}+\int_{K_{F}}\left[\left(\nabla w_{h \mid K}-\nabla v_{h \mid K_{F}}\right) \cdot\left(\boldsymbol{x}-\overline{\boldsymbol{x}}_{F}\right)\right]^{2} \\
& :=\mathfrak{T}_{2,1}+\mathfrak{T}_{2,2} .
\end{aligned}
$$

Using (2), the Cauchy-Schwarz inequality, and Proposition 7 it is inferred that

$$
\mathfrak{T}_{2,1}=\frac{|F| d_{K, F}}{d}\left(\left\langle w_{h \mid K}-v\right\rangle_{F}\right)^{2} \leqslant \frac{d_{K, F}}{d}\left\|w_{h \mid K}-v\right\|_{L^{2}(F)}^{2} \lesssim h_{K}^{2(l+1)}|v|_{H^{l+1}(K)}^{2} .
$$

Since $\left|\boldsymbol{x}-\overline{\boldsymbol{x}}_{F}\right| \leqslant h_{K}$ for all $\boldsymbol{x} \in K_{F}$ and both $\boldsymbol{\nabla} w_{h \mid K}$ and $\boldsymbol{\nabla} v_{h \mid K_{F}}$ are constant on $K_{F}$, the estimate (19) yields

$$
\mathfrak{T}_{2,2} \leqslant h_{K}^{2}\left\|\nabla w_{h \mid K}-\nabla v_{h \mid K_{F}}\right\|_{L^{2}\left(K_{F}\right)^{d}}^{2} \lesssim h_{K}^{2(l+1)}|v|_{H^{l+1}(K)}^{2} .
$$

Summing over $F \in \mathcal{F}_{K}$, using the bounds for $\mathfrak{T}_{2,1}$ and $\mathfrak{T}_{2,2}$ together with the fact that $\operatorname{card}\left(\mathcal{F}_{K}\right)$ is bounded uniformly in $h$, it is inferred that $\mathfrak{T}_{2} \lesssim h_{K}^{l+1}|v|_{H^{l+1}(K)}$, thereby yielding $\left\|v-v_{h}\right\|_{L^{2}(K)} \lesssim h_{K}^{l+1}|v|_{H^{l+1}(K)}$, and therefore concluding the proof.

Remark 11 (The matching simplicial case). When $\mathcal{K}_{h}$ is matching simplicial, the proof of Lemma 10 can be simplified exploiting the result of Proposition 9 to infer

$$
\inf _{v_{h} \in \mathfrak{C} \mathfrak{R}\left(\mathcal{K}_{h}\right)}\left\|v-v_{h}\right\|_{H^{1}(K)} \leqslant \inf _{v_{h} \in \mathbb{C} \mathbb{R}\left(\mathcal{K}_{h}\right)}\left\|v-v_{h}\right\|_{H^{1}(K)},
$$

and conclude using the approximation properties of the standard Crouzeix-Raviart space.

For all $\boldsymbol{w} \in \boldsymbol{H}\left(\operatorname{div} ; \mathcal{P}_{h}\right):=\left\{\boldsymbol{v} \in L^{2}(\Omega)^{d} \mid \forall P \in \mathcal{P}_{h}, \nabla \cdot\left(\boldsymbol{v}_{\mid P}\right) \in L^{2}(P)\right\}$ let

$$
D_{h}(\boldsymbol{w}):=\Pi_{h}^{0}\left(\nabla_{h} \cdot \boldsymbol{w}\right) .
$$

An immediate consequence of the first point in Lemma 10 is that the discrete vector space $\mathfrak{C} \mathfrak{R}\left(\mathcal{K}_{h}\right)^{d}$ possesses the following approximation property.

Corollary 12 (Divergence approximation). Let $\boldsymbol{v} \in H^{1}(\Omega)^{d}$ and $\boldsymbol{v}_{h}:=\mathcal{I}_{h}^{\mathfrak{C} \Re}(\boldsymbol{v})$. There holds

$$
D_{h}\left(\boldsymbol{v}_{h}\right)=\Pi_{h}^{0}(\boldsymbol{\nabla} \cdot \boldsymbol{v})
$$


Moreover, there exists a real $C>0$ independent of the meshsize such that, for all $h \in \mathcal{H}$, all $K \in \mathcal{K}_{h}$, and all $\boldsymbol{v} \in H^{1}(\Omega)^{d} \cap \boldsymbol{H}^{1}\left(\operatorname{div} ; \mathcal{K}_{h}\right)$ with

$$
\boldsymbol{H}^{1}\left(\operatorname{div} ; \mathcal{K}_{h}\right):=\left\{\boldsymbol{v} \in \boldsymbol{H}(\operatorname{div} ; \Omega) \mid \nabla_{h} \cdot \boldsymbol{v} \in H^{1}\left(\mathcal{K}_{h}\right)\right\}
$$

and $\boldsymbol{v}_{h}:=\mathcal{I}_{h}^{\mathfrak{C} R}(\boldsymbol{v})$,

$$
\left\|\boldsymbol{\nabla} \cdot \boldsymbol{v}-D_{h}\left(\boldsymbol{v}_{h}\right)\right\|_{L^{2}(K)}+h_{K}\left|\boldsymbol{\nabla} \cdot \boldsymbol{v}-D_{h}\left(\boldsymbol{v}_{h}\right)\right|_{H^{1}(K)} \leqslant C h_{K}|\boldsymbol{\nabla} \cdot \boldsymbol{v}|_{H^{1}(K)} .
$$

Thus, according to Corollary 12 and Lemma 10 (which gives an $H^{1}$-stability property), $\mathcal{I}_{h}^{\mathfrak{C} R}$ can play the role of a Fortin operator [10] for the Stokes problem; see the proof of Lemma 26

\section{Applications}

In this section we present two applications where the properties of the discrete space (11) are instrumental in designing a suitable numerical approximation, namely linear elasticity for quasi-incompressible materials and the Stokes problem.

4.1. Discrete $H_{0}^{1}$-norm. For problems naturally set in $H_{0}^{1}(\Omega)$, boundary conditions can be accounted for in a strong manner by introducing the following subspace of $\mathfrak{C} \Re\left(\mathcal{K}_{h}\right)$ :

$$
\mathfrak{C} \mathfrak{R}_{0}\left(\mathcal{K}_{h}\right):=\mathfrak{R}_{h}\left(\mathbb{V}_{h, 0}\right), \quad \mathbb{V}_{h, 0}=\left\{\mathbb{}_{h} \in \mathbb{V}_{h} \mid v_{F}=0, \forall F \in \mathcal{F}_{\mathcal{K}_{h}}^{\mathrm{b}}\right\} .
$$

In the following proposition we show that the $L^{2}$-norm of the broken gradient is a norm on $\mathfrak{C} \mathfrak{R}_{0}\left(\mathcal{K}_{h}\right)$ by proving uniform discrete equivalence with the usual dG norm; cf. [17, Section 5.1]:

$$
\left\|v_{h}\right\|_{\mathrm{dG}}^{2}:=\left\|\nabla_{h} v_{h}\right\|_{L^{2}(\Omega)^{d}}^{2}+\left|v_{h}\right|_{\mathrm{J}}^{2}, \quad\left|v_{h}\right|_{\mathrm{J}}^{2}:=\sum_{F \in \mathcal{F}_{\mathcal{P}_{h}}} \frac{1}{h_{F}}\left\|\llbracket v_{h} \rrbracket\right\|_{L^{2}(F)}^{2} .
$$

Proposition 13 (Discrete norm). Assume $\eta=d$ in (9). Then, there exists a real $C>0$ independent of the meshsize such that, for all $v_{h} \in \mathfrak{C} \mathfrak{R}_{0}\left(\mathcal{K}_{h}\right)$,

$$
\left\|\nabla_{h} v_{h}\right\|_{L^{2}(\Omega)^{d}} \leqslant\left\|v_{h}\right\|_{\mathrm{dG}} \leqslant C\left\|\boldsymbol{\nabla}_{h} v_{h}\right\|_{L^{2}(\Omega)^{d}} .
$$

Proof. The notation $a \lesssim b$ stands for $a \leqslant C b$ with $C>0$ independent of the meshsize. Clearly, $\left\|\nabla_{h} v_{h}\right\|_{L^{2}(\Omega)^{d}} \leqslant\left\|v_{h}\right\|_{\mathrm{dG}}$ for all $v_{h} \in \mathfrak{C} \mathfrak{R}_{0}\left(\mathcal{K}_{h}\right)$. To prove that $\left\|v_{h}\right\|_{\mathrm{dG}} \lesssim\left\|\boldsymbol{\nabla}_{h} v_{h}\right\|_{L^{2}(\Omega)^{d}}$ for all $v_{h} \in \mathfrak{C}_{0}\left(\mathcal{K}_{h}\right)$, it suffices to show that $\left|v_{h}\right|_{\mathrm{J}} \lesssim$ $\left\|\nabla_{h} v_{h}\right\|_{L^{2}(\Omega)^{d}}$. Let $P \in \mathcal{P}_{h}$ and $F \in \mathcal{F}_{P}$. Owing to the linearity of $v_{h}$ inside $P$ there holds for all $\boldsymbol{x} \in P, v_{h \mid P}(\boldsymbol{x})=\left\langle v_{h \mid P}\right\rangle_{F}+\boldsymbol{\nabla} v_{h \mid P} \cdot\left(\boldsymbol{x}-\overline{\boldsymbol{x}}_{F}\right)$. This together with Lemma 8 and the discrete trace inequality (5) yields

$$
\begin{aligned}
\left\|\llbracket v_{h} \rrbracket\right\|_{L^{2}(F)} & =\left\|\llbracket v_{h} \rrbracket-\left\langle\llbracket v_{h} \rrbracket\right\rangle_{F}\right\|_{L^{2}(F)} \leqslant h_{F}\left\|\llbracket \nabla_{h} v_{h} \rrbracket\right\|_{L^{2}(F)^{d}} \\
& \lesssim h_{F}^{1 / 2} \sum_{P \in \mathcal{P}_{F}}\left\|\nabla v_{h \mid P}\right\|_{L^{2}(P)^{d}},
\end{aligned}
$$

where we have set $\mathcal{P}_{F}:=\left\{P \in \mathcal{P}_{h} \mid F \subset \partial P\right\}$. Using (22) together with (23) and the Cauchy-Schwarz inequality it is inferred that

$$
\begin{aligned}
\left|v_{h}\right|_{\mathrm{J}}^{2} & =\sum_{F \in \mathcal{F}_{\mathcal{P}_{h}}} \frac{1}{h_{F}}\left\|\llbracket v_{h} \rrbracket-\left\langle\llbracket v_{h} \rrbracket\right\rangle_{F}\right\|_{L^{2}(F)}^{2} \\
& \lesssim \sum_{F \in \mathcal{F}_{\mathcal{P}_{h}}} \sum_{P \in \mathcal{P}_{F}}\left\|\nabla v_{h \mid P}\right\|_{L^{2}(P)^{d}}^{2} \lesssim\left\|\nabla_{h} v_{h}\right\|_{L^{2}(\Omega)^{d}}^{2},
\end{aligned}
$$


where the last bound is a consequence of the fact that the maximum number of faces of a pyramid is bounded uniformly in $h$ since $\mathcal{P}_{h}$ is shape- and contact-regular; cf. [17, Lemma 1.41].

4.2. Quasi-incompressible linear elasticity. We tackle here the question of the accurate approximation of the linear elasticity equations in the quasi-incompressible limit on general polygonal or polyhedral meshes. The main sources of inspiration are the classical works of Brenner and Sung [9, and Hansbo and Larson [28, 29].

4.2.1. Setting. We consider a homogeneous elastic material occupying the polygonal/polyhedral domain $\Omega \subset \mathbb{R}^{d}$, and whose mechanical properties are described by the real Lamé parameters $\lambda$ and $\mu$ with $0<\mu<+\infty$ and $0<\lambda \leqslant+\infty$. The mechanical behavior of the material is governed by the linear elasticity equations,

$$
\begin{aligned}
-\nabla \cdot \underline{\sigma}(\boldsymbol{u}) & =\boldsymbol{f} & & \text { in } \Omega, \\
\boldsymbol{u} & =\mathbf{0} & & \text { on } \partial \Omega,
\end{aligned}
$$

where $\boldsymbol{u}$ denotes the vector-valued displacement field, $\boldsymbol{f} \in L^{2}(\Omega)^{d}$ the forcing term, and the Cauchy stress tensor is defined for all $\boldsymbol{v} \in H^{1}(\Omega)^{d}$ by Hooke's law,

$$
\underline{\underline{\sigma}}(\boldsymbol{v}):=2 \mu \underline{\underline{\varepsilon}}(\boldsymbol{v})+\lambda \boldsymbol{\nabla} \cdot \underline{\underline{v}} \underline{\underline{I_{d}}}, \quad \underline{\underline{\varepsilon}}(\boldsymbol{v}):=\frac{1}{2}\left(\boldsymbol{\nabla v}+\boldsymbol{\nabla} \boldsymbol{v}^{T}\right) .
$$

Setting $\boldsymbol{U}:=H_{0}^{1}(\Omega)^{d}$, the weak formulation of problem reads: Find $\boldsymbol{u} \in \boldsymbol{U}$ such that

$$
\tilde{a}(\boldsymbol{u}, \boldsymbol{v})=(\boldsymbol{f}, \boldsymbol{v})_{L^{2}(\Omega)^{d}} \quad \forall \boldsymbol{v} \in \boldsymbol{U},
$$

where $\tilde{a}(\boldsymbol{u}, \boldsymbol{v}):=(\underline{\underline{\sigma}}(\boldsymbol{u}), \underline{\underline{\varepsilon}}(\boldsymbol{v}))_{L^{2}(\Omega)^{d, d}}$. The well-posedness of the weak formulation (26) relies on Korn's inequality in $\boldsymbol{U}$ (cf., e.g., [1, Lemma 5.3.2]),

$$
\|\boldsymbol{\nabla} \boldsymbol{v}\|_{L^{2}(\Omega)^{d, d}} \leqslant \sqrt{2}\|\underline{\underline{\varepsilon}}(\boldsymbol{v})\|_{L^{2}(\Omega)^{d, d}} \quad \forall \boldsymbol{v} \in \boldsymbol{U} .
$$

The use of Korn's inequality can be circumvented for the pure displacement problem using the following alternative Navier-Cauchy formulation: Find $\boldsymbol{u} \in \boldsymbol{U}$ such that $(28)$

$a(\boldsymbol{u}, \boldsymbol{v}):=(\mu \boldsymbol{\nabla} \boldsymbol{u}, \boldsymbol{\nabla} \boldsymbol{v})_{L^{2}(\Omega)^{d, d}}+((\mu+\lambda) \boldsymbol{\nabla} \cdot \boldsymbol{u}, \boldsymbol{\nabla} \cdot \boldsymbol{v})_{L^{2}(\Omega)}=(\boldsymbol{f}, \boldsymbol{v})_{L^{2}(\Omega)^{d}} \quad \forall \boldsymbol{v} \in \boldsymbol{U}$.

This naturally coercive formulation is equivalent to (26) for homogeneous materials and pure Dirichlet boundary conditions. Throughout the rest of this section we focus on this alternative form. The treatment of mixed-type boundary conditions is addressed in Section 4.2.5 We recall the following regularity result valid in $d=2$; cf., e.g., [9] Lemma 2.2].

Lemma 14 (Regularity). Let $d=2$ and assume that $\Omega$ is convex. Then, problem (25) has a unique solution $\boldsymbol{u} \in \boldsymbol{U}_{*}:=\boldsymbol{U} \cap H^{2}(\Omega)^{d}$. Moreover, there exists a real $C_{\mu}>0$ only depending on $\Omega$ and $\mu$ but not on $\lambda$, such that, for $\lambda$ large enough,

$$
\mathcal{N}_{\mathrm{el}}(\boldsymbol{u}):=\|\boldsymbol{u}\|_{H^{2}(\Omega)^{d}}+|\lambda \boldsymbol{\nabla} \cdot \boldsymbol{u}|_{H^{1}(\Omega)} \leqslant C_{\mu}\|\boldsymbol{f}\|_{L^{2}(\Omega)^{d}} .
$$

This implies, in particular, that, if $\lambda \rightarrow+\infty$, the divergence of the displacement field approaches zero, corresponding to a quasi-incompressible material; cf. [19] for a generalization to composite materials. 
4.2.2. Discretization. Let $\mathcal{K}_{h}$ belong to an admissible mesh sequence in the sense of Definition 4. We consider an approximation of the displacement field in the space

$$
\boldsymbol{U}_{h}:=\mathfrak{C}^{\mathfrak{r}}\left(\mathcal{K}_{h}\right)^{d}
$$

with $\mathfrak{C R}_{0}\left(\mathcal{K}_{h}\right)$ defined by (21). Henceforth we assume the choice $\eta=d$ in (9), so that the continuity of mean values stated in Lemma 8 holds. Lowest-order, locking-free discretizations of (25) satisfy an estimate of the form

$$
\left\|\boldsymbol{u}-\boldsymbol{u}_{h}\right\|_{\mathrm{el}} \leqslant C h\|\boldsymbol{f}\|_{L^{2}(\Omega)^{d}},
$$

where $\|\cdot\|_{\mathrm{el}}$ is a (discrete) energy norm and $C>0$ is a constant possibly depending on $\mu$ and on the mesh regularity parameters but independent of $h, \lambda$, and $\boldsymbol{u}$. The key point is here that the multiplicative constant in the right-hand side of (30) does not blow up in the limit $\lambda \rightarrow+\infty$, i.e., the method converges uniformly in $\lambda$. To obtain (30) we prove that in general space dimension there holds with $\mathcal{N}_{\text {el }}(\boldsymbol{u})$ defined by (29),

$$
\left\|\boldsymbol{u}-\boldsymbol{u}_{h}\right\|_{\mathrm{el}} \leqslant C_{\mathrm{el}} h \mathcal{N}_{\mathrm{el}}(\boldsymbol{u}),
$$

where $C_{\mathrm{el}}>0$ has the same dependencies as $C$, and then invoke the regularity estimate in Lemma 14 to conclude in $d=2$. The discrete problem reads: Find $\boldsymbol{u}_{h} \in \boldsymbol{U}_{h}$ such that

$$
a_{h}\left(\boldsymbol{u}_{h}, \boldsymbol{v}_{h}\right)=\left(\boldsymbol{f}, \boldsymbol{v}_{h}\right)_{L^{2}(\Omega)^{d}} \quad \forall \boldsymbol{v}_{h} \in \boldsymbol{U}_{h},
$$

with discrete bilinear form $a_{h}$ such that

$$
\begin{aligned}
a_{h}(\boldsymbol{w}, \boldsymbol{v}):= & \left(\mu \boldsymbol{\nabla}_{h} \boldsymbol{w}, \boldsymbol{\nabla}_{h} \boldsymbol{v}\right)_{L^{2}(\Omega)^{d, d}}+\left(\mu \boldsymbol{\nabla}_{h} \cdot \boldsymbol{w}, \boldsymbol{\nabla}_{h} \cdot \boldsymbol{v}\right)_{L^{2}(\Omega)} \\
& +\left(\lambda D_{h}(\boldsymbol{w}), D_{h}(\boldsymbol{v})\right)_{L^{2}(\Omega)} .
\end{aligned}
$$

The last term in the right-hand side is treated using the discrete divergence $D_{h}$ defined in Corollary 12 since the approximation properties of $D_{h}$ are instrumental to ensure that $\lambda$ only appears in terms of the form $|\lambda \boldsymbol{\nabla} \cdot \boldsymbol{u}|_{H^{1}(\Omega)}$ in the right-hand side of the error estimate (cf., in particular, the bound for the consistency term in the proof of Theorem [18).

Remark 15 (Implementation). In (31), cell-centered unknowns for a given element $K \in \mathcal{K}_{h}$ are only linked with the face unknowns located on the boundary of $K$. As a result, they can be locally eliminated by taking the Schur complement of the corresponding block in the local matrix. This requires, in general, to inverse a $d \times d$ matrix. However, this cost can be further reduced by replacing in (32) the term $\left(\mu \boldsymbol{\nabla}_{h} \cdot \boldsymbol{u}_{h}, \boldsymbol{\nabla}_{h} \cdot \boldsymbol{v}_{h}\right)_{L^{2}(\Omega)}$ by $\left(\mu D_{h}\left(\boldsymbol{u}_{h}\right), D_{h}\left(\boldsymbol{v}_{h}\right)\right)_{L^{2}(\Omega)}$. This choice avoids, without jeopardizing the approximation, the interaction of the cell unknowns for the different components of the displacement, hence the corresponding block of the local matrix is diagonal and trivial to invert.

The energy norm associated to the bilinear form $a_{h}$ is

$$
\|\boldsymbol{v}\|_{\mathrm{el}}^{2}:=a_{h}(\boldsymbol{v}, \boldsymbol{v})=\left\|\mu^{1 / 2} \nabla_{h} \boldsymbol{v}\right\|_{L^{2}(\Omega)^{d, d}}^{2}+\left\|\mu^{1 / 2} \nabla_{h} \cdot \boldsymbol{v}\right\|_{L^{2}(\Omega)}^{2}+\left\|\lambda^{1 / 2} D_{h}(\boldsymbol{v})\right\|_{L^{2}(\Omega)}^{2} .
$$

Lemma 16 (Coercivity). There holds for all $\boldsymbol{v}_{h} \in \boldsymbol{U}_{h}$,

$$
a_{h}\left(\boldsymbol{v}_{h}, \boldsymbol{v}_{h}\right)=\left\|\boldsymbol{v}_{h}\right\|_{\mathrm{el}}^{2} \geqslant \mu\left\|\nabla_{h} \boldsymbol{v}_{h}\right\|_{L^{2}(\Omega)^{d, d}}^{2} .
$$


The well-posedness of the discrete problem (31) then follows from the LaxMilgram Lemma and Proposition 13 Now let $\boldsymbol{U}_{* h}:=\boldsymbol{U}_{*}+\boldsymbol{U}_{h}$ with $\boldsymbol{U}_{*}$ defined in Lemma 14, and extend the bilinear form $a_{h}$ to $\boldsymbol{U}_{* h} \times \boldsymbol{U}_{* h}$. Assuming $\boldsymbol{u} \in \boldsymbol{U}_{*}$ ensures that the boundary terms in the expression of the consistency error are well-defined; cf. Lemma 17. This additional regularity is verified, e.g., under the assumptions of Lemma 14

Lemma 17 (Weak consistency). Let $\boldsymbol{u} \in \boldsymbol{U}$ denote the solution to (28) and additionally assume $\boldsymbol{u} \in \boldsymbol{U}_{*}$. Then, there holds for all $\boldsymbol{v}_{h} \in \boldsymbol{U}_{h}$,

$$
a_{h}\left(\boldsymbol{u}, \boldsymbol{v}_{h}\right)=\left(\boldsymbol{f}, \boldsymbol{v}_{h}\right)_{L^{2}(\Omega)^{d}}+\mathcal{E}_{h}\left(\boldsymbol{v}_{h}\right),
$$

where, letting $\underline{\underline{\tau}}(\boldsymbol{u}):=\mu \boldsymbol{\nabla} \boldsymbol{u}+(\mu+\lambda)(\boldsymbol{\nabla} \cdot \boldsymbol{u}) \underline{\underline{I_{d}}}$,

$$
\mathcal{E}_{h}\left(\boldsymbol{v}_{h}\right):=\sum_{F \in \mathcal{F}_{\mathcal{P}_{h}}}\left(\underline{\underline{\tau}}(\boldsymbol{u}) \boldsymbol{n}_{F}, \llbracket \boldsymbol{v}_{h} \rrbracket\right)_{L^{2}(F)^{d}}+\left(\lambda\left(D_{h}(\boldsymbol{u})-\boldsymbol{\nabla} \cdot \boldsymbol{u}\right), \boldsymbol{\nabla}_{h} \cdot \boldsymbol{v}_{h}\right)_{L^{2}(\Omega)} .
$$

Proof. Observing that $\left.\left(\lambda D_{h}(\boldsymbol{u}), D_{h}\left(\boldsymbol{v}_{h}\right)\right)_{L^{2}(\Omega)}=\left(\lambda D_{h}(\boldsymbol{u}), \nabla_{h} \cdot \boldsymbol{v}_{h}\right)\right)_{L^{2}(\Omega)}$, and summing and subtracting $\left(\lambda \boldsymbol{\nabla} \cdot \boldsymbol{u}, \boldsymbol{\nabla}_{h} \cdot \boldsymbol{v}_{h}\right)_{L^{2}(\Omega)}$ from the right-hand side of (32) with $(\boldsymbol{w}, \boldsymbol{v})=\left(\boldsymbol{u}, \boldsymbol{v}_{h}\right)$ yields

$$
a_{h}\left(\boldsymbol{u}, \boldsymbol{v}_{h}\right)=\left(\underline{\underline{\tau}}(\boldsymbol{u}), \nabla_{h} \boldsymbol{v}_{h}\right)_{L^{2}(\Omega)^{d, d}}+\left(\lambda\left(D_{h}(\boldsymbol{u})-\boldsymbol{\nabla} \cdot \boldsymbol{u}\right), \nabla_{h} \cdot \boldsymbol{v}_{h}\right)_{L^{2}(\Omega)} .
$$

Integrating by parts the first term elementwise, rearranging the boundary contributions, and using $\llbracket \underline{\underline{\tau}}(\boldsymbol{u}) \rrbracket_{F} \boldsymbol{n}_{F}=\mathbf{0}$ and $\{\underline{\underline{\tau}}(\boldsymbol{u})\}_{F} \boldsymbol{n}_{F}=\underline{\underline{\tau}}(\boldsymbol{u}) \boldsymbol{n}_{F}$ for all $F \in \mathcal{F}_{\mathcal{P}_{h}}^{\mathrm{i}}$, it is inferred that

$$
\begin{aligned}
(\underline{\underline{\tau}}(\boldsymbol{u}), & \left.\boldsymbol{\nabla}_{h} \boldsymbol{v}_{h}\right)_{L^{2}(\Omega)^{d, d}}=-\left(\boldsymbol{\nabla} \cdot \underline{\underline{\tau}}(\boldsymbol{u}), \boldsymbol{v}_{h}\right)_{L^{2}(\Omega)^{d}}+\sum_{P \in \mathcal{P}_{h}}\left(\underline{\underline{\tau}}(\boldsymbol{u}) \boldsymbol{n}_{P}, \boldsymbol{v}_{h \mid P}\right)_{L^{2}(\partial P)^{d}} \\
= & \left(\boldsymbol{f}, \boldsymbol{v}_{h}\right)_{L^{2}(\Omega)^{d}}+\sum_{F \in \mathcal{F}_{\mathcal{P}_{h}}^{\mathrm{i}}}\left(\llbracket \underline{\underline{\tau}}(\boldsymbol{u}) \rrbracket \boldsymbol{n}_{F},\left\{\boldsymbol{v}_{h}\right\}\right)_{L^{2}(F)^{d}} \\
& +\sum_{F \in \mathcal{F}_{\mathcal{P}_{h}}}\left(\{\underline{\underline{\tau}}(\boldsymbol{u})\} \boldsymbol{n}_{F}, \llbracket \boldsymbol{v}_{h} \rrbracket\right)_{L^{2}(F)^{d}} \\
= & \left(\boldsymbol{f}, \boldsymbol{v}_{h}\right)_{L^{2}(\Omega)^{d}}+\sum_{F \in \mathcal{F}_{\mathcal{P}_{h}}}\left(\underline{\underline{\tau}}(\boldsymbol{u}) \boldsymbol{n}_{F}, \llbracket \boldsymbol{v}_{h} \rrbracket\right)_{L^{2}(F)^{d}}
\end{aligned}
$$

where we have used $-\boldsymbol{\nabla} \cdot \underline{\underline{\tau}}(\boldsymbol{u})=\boldsymbol{f}$ a.e. in $\Omega$ in the second line (cf. (28)). This concludes the proof.

In the following theorem the continuity of mean values at interfaces plays an important role in estimating the boundary contribution in the consistency error.

Theorem 18 (Error estimate for (31)). Let $\boldsymbol{u} \in \boldsymbol{U}$ denote the solution to (28) and additionally assume $\boldsymbol{u} \in \boldsymbol{U}_{*}$. Then, there exists $C_{\mathrm{el}}>0$ independent of the meshsize, of $\lambda$, and of $\boldsymbol{u}$ such that, denoting by $\boldsymbol{u}_{h} \in \boldsymbol{U}_{h}$ the solution to (31), there holds

$$
\left\|\boldsymbol{u}-\boldsymbol{u}_{h}\right\|_{\mathrm{el}} \leqslant C_{\mathrm{el}} h \mathcal{N}_{\mathrm{el}}(\boldsymbol{u})
$$


Proof. We note $a \lesssim b$ the inequality $a \leqslant C b$ where $C>0$ has the same dependence as the constant $C_{\text {el }}$ in (35). The Cauchy-Schwarz inequality yields boundedness in the form $a_{h}(\boldsymbol{w}, \boldsymbol{v}) \leqslant\|\boldsymbol{w}\|_{\text {el }}\|\boldsymbol{v}\|_{\text {el }}$ for all $(\boldsymbol{w}, \boldsymbol{v}) \in \boldsymbol{U}_{* h} \times \boldsymbol{U}_{* h}$. This, together with Lemmas 16 and 17 and the Second Strang Lemma [32] (cf. also [24, Lemma 2.25]), yields:

$$
\left\|\boldsymbol{u}-\boldsymbol{u}_{h}\right\|_{\mathrm{el}} \lesssim \inf _{\boldsymbol{v}_{h} \in \boldsymbol{U}_{h}}\left\|\boldsymbol{u}-\boldsymbol{v}_{h}\right\|_{\mathrm{el}}+\sup _{\boldsymbol{v}_{h} \in \boldsymbol{U}_{h} \backslash\{\mathbf{0}\}} \frac{\left|\mathcal{E}_{h}\left(\boldsymbol{v}_{h}\right)\right|}{\left\|\boldsymbol{v}_{h}\right\|_{\mathrm{el}}}:=\mathfrak{T}_{1}+\mathfrak{T}_{2} .
$$

The first term in the right-hand side depends on the approximation properties of the discrete space in the $\|\cdot\|_{\mathrm{el}}$-norm, while the second is linked to the consistency error. Let $\boldsymbol{w}_{h}:=\mathcal{I}_{h}^{\mathfrak{C} \Re}(\boldsymbol{u}) \in \boldsymbol{U}_{h}$. Using Lemma 10 twice and Corollary 12 respectively to treat the three terms in the right-hand side of (33) with $\boldsymbol{v}=\boldsymbol{u}-\boldsymbol{w}_{h}$ we infer,

$$
\mathfrak{T}_{1} \leqslant\left\|\boldsymbol{u}-\boldsymbol{w}_{h}\right\|_{\mathrm{el}} \lesssim h\|\boldsymbol{u}\|_{H^{2}(\Omega)^{d}}+h\left|\lambda^{1 / 2} \nabla \cdot \boldsymbol{u}\right|_{H^{1}(\Omega)} .
$$

To treat the consistency error, denote by $\mathfrak{T}_{2,1}$ and $\mathfrak{T}_{2,2}$ the two terms in the righthand side of (34). Let

$$
\underline{\underline{\phi_{\mu}}}:=\mu\left(\boldsymbol{\nabla} \boldsymbol{u}-\Pi_{h}^{0}(\boldsymbol{\nabla} \boldsymbol{u})\right), \quad \psi_{s}:=s\left(\boldsymbol{\nabla} \cdot \boldsymbol{u}-\Pi_{h}^{0}(\boldsymbol{\nabla} \cdot \boldsymbol{u})\right), \text { with } s \in\{\mu, \lambda\} .
$$

Clearly, $\psi_{\mu}=\operatorname{tr}\left(\underline{\underline{\phi_{\mu}}}\right)$. Using the continuity of mean values at interfaces together with the fact that both $\left\{\mu \Pi_{h}^{0}(\boldsymbol{\nabla} \boldsymbol{u})\right\}_{F}$ and $\left\{s \Pi_{h}^{0}(\boldsymbol{\nabla} \cdot \boldsymbol{u})\right\}_{F}, s \in\{\mu, \lambda\}$, are constant on every $F \in \mathcal{F}_{\mathcal{P}_{h}}$, it is inferred that

$$
\begin{aligned}
\mathfrak{T}_{2,1}= & \sum_{F \in \mathcal{F}_{\mathcal{P}_{h}}}\left(\left\{\underline{\underline{\tau}}(\boldsymbol{u})-\Pi_{h}^{0}(\underline{\underline{\tau}}(\boldsymbol{u}))\right\} \boldsymbol{n}_{F}, \llbracket \boldsymbol{v}_{h} \rrbracket-\left\langle\llbracket \boldsymbol{v}_{h} \rrbracket\right\rangle_{F}\right)_{L^{2}(F)^{d}} \\
= & \sum_{F \in \mathcal{F}_{\mathcal{P}_{h}}}\left(\left\{\underline{\underline{\phi_{\mu}}}+\psi_{\mu} \underline{\underline{I_{d}}}\right\} \boldsymbol{n}_{F}, \llbracket \boldsymbol{v}_{h} \rrbracket-\left\langle\llbracket \boldsymbol{v}_{h} \rrbracket\right\rangle_{F}\right)_{L^{2}(F)^{d}} \\
& +\sum_{F \in \mathcal{F}_{\mathcal{P}_{h}}}\left(\left\{\psi_{\lambda}\right\} \boldsymbol{n}_{F}, \llbracket \boldsymbol{v}_{h} \rrbracket-\left\langle\llbracket \boldsymbol{v}_{h} \rrbracket\right\rangle_{F}\right)_{L^{2}(F)^{d}} .
\end{aligned}
$$

The Cauchy-Schwarz inequality followed by the trace inequality (6) with $\mathcal{S}_{h}=\mathcal{P}_{h}$, the fact that the maximum number of faces of a pyramid is bounded uniformly in $h$, the approximation properties of the $L^{2}$-orthogonal projection, and (24) yield

$$
\begin{aligned}
\mathfrak{T}_{2,1} \lesssim & \left\{\sum_{P \in \mathcal{P}_{h}} h\left(\left\|\underline{\underline{\phi_{\mu}}} \mid P\right\|_{L^{2}(\partial P)^{d, d}}^{2}+\left\|\psi_{\lambda \mid P}\right\|_{L^{2}(\partial P)}^{2}\right)\right\}^{\frac{1}{2}} \\
& \times\left\{\sum_{F \in \mathcal{F}_{\mathcal{P}_{h}}} h_{F}^{-1}\left\|\llbracket \boldsymbol{v}_{h} \rrbracket-\left\langle\llbracket \boldsymbol{v}_{h} \rrbracket\right\rangle_{F}\right\|_{L^{2}(F)^{d}}^{2}\right\}^{\frac{1}{2}} \\
\lesssim & h \mathcal{N}_{\mathrm{el}}(\boldsymbol{u})\left\|\boldsymbol{v}_{h}\right\|_{\mathrm{el}},
\end{aligned}
$$

where the bound $\left\|\boldsymbol{\nabla}_{h} \boldsymbol{v}_{h}\right\|_{L^{2}(\Omega)^{d, d}} \lesssim\left\|\boldsymbol{v}_{h}\right\|_{\text {el }}$ is a consequence of Lemma 16] Finally, using the Cauchy-Schwarz inequality together with the approximation properties of the $L^{2}$-orthogonal projection and Lemma 16 it is inferred that

$$
\mathfrak{T}_{2,2} \leqslant\left\|\lambda\left(\Pi_{h}^{0}(\boldsymbol{\nabla} \cdot \boldsymbol{u})-\boldsymbol{\nabla} \cdot \boldsymbol{u}\right)\right\|_{L^{2}(\Omega)}\left\|\boldsymbol{\nabla}_{h} \cdot \boldsymbol{v}_{h}\right\|_{L^{2}(\Omega)} \lesssim h|\lambda \boldsymbol{\nabla} \cdot \boldsymbol{u}|_{H^{1}(\Omega)}\left\|\boldsymbol{v}_{h}\right\|_{\mathrm{el}} \cdot
$$

Using inequalities (37), (38), and (39) to bound the right-hand side of (36) the conclusion follows. 


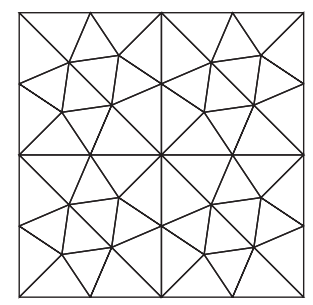

(A) Triangular

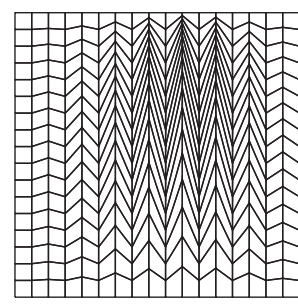

(B) Kershaw

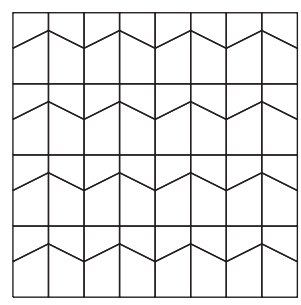

(c) Trapezoidal

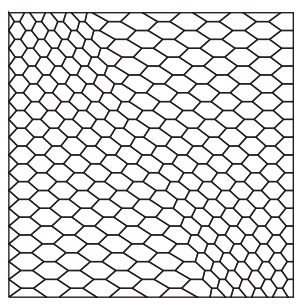

(D) Hexagonal

Figure 3. Members of the mesh families for the numerical test of Section 4.2.3

Corollary 19 (Uniform convergence with respect to $\lambda$ in $d=2$ ). If $d=2$ the locking-free error estimate (30) holds with $C=C_{\mathrm{el}} C_{\mu}$.

Remark 20 (Use of Lemma 14). In the proof of Theorem 18, the a priori bound on $|\lambda \boldsymbol{\nabla} \cdot \boldsymbol{u}|_{H^{1}(\Omega)}$ is only required to bound $\mathfrak{T}_{2}$. For $\mathfrak{T}_{1}$, the weaker regularity estimate $\|\boldsymbol{u}\|_{H^{2}(\Omega)^{d}}+\left|\lambda^{1 / 2} \boldsymbol{\nabla} \cdot \boldsymbol{u}\right|_{H^{1}(\Omega)} \lesssim\|\boldsymbol{f}\|_{L^{2}(\Omega)^{d}}$ is sufficient.

Remark 21 ( $L^{2}$-error estimate). Optimal error estimates for the $L^{2}$-error on the displacement can be derived using the Aubin-Nitsche trick based on the symmetry of the method.

4.2.3. A numerical example: the closed cavity problem. To assess the robustness of the method (31) in the quasi-incompressible limit, we consider the closed cavity problem of Hansbo and Larson [29. Although this problem does not exhibit the regularity required by Theorem 18] it is included as it is one of the simplest benchmarks for numerical locking. The implementation relies on the general framework recently introduced in 18 , to which we refer for further details.

We let $\Omega:=(0,1)^{2}, \boldsymbol{f} \equiv \mathbf{0}$, and prescribe a horizontal displacement $\boldsymbol{u}=(1,0)$ on the upper side and $\boldsymbol{u}=(0,0)$ on the remaining three. The elastic modulus and Poisson's ratio are chosen as $E=1000$ and $\nu=0.4999$ respectively. The Lamé parameters can be derived from the relations $\lambda=\frac{\nu E}{(1+\nu)(1-2 \nu)}, \mu=\frac{E}{2(1+\nu)}$, which show, in particular, that $\lambda \rightarrow+\infty$ as $\nu \rightarrow 1 / 2$. The discrete problem (31) is solved on the four mesh sequences depicted in Figure 3 From each mesh family, a coarse and a fine mesh are selected featuring roughly the same number of elements. Figure 4 depicts the values of the horizontal displacement $u_{h, 1}$ along the vertical centerline $x_{1}=1 / 2$ as well as the values of the vertical displacement $u_{h, 2}$ along the horizontal centerline $x_{2}=1 / 2$. The results of the (more expensive) dG method of [19] on the matching triangular mesh are also included for comparison. As predicted, no sign of numerical locking is observed.

\subsubsection{Variations and links with finite volume and finite element methods.}

Flux formulation and local conservation. Following [25, Section 2.4] it is possible to reformulate the discrete bilinear form (32) in terms of numerical fluxes. More specifically, introducing $\mathbb{U}_{h}:=\mathbb{V}_{h, 0}^{d}$ where $\mathbb{V}_{h, 0}$ is defined by (21), let $\boldsymbol{w}_{h}, \boldsymbol{v}_{h} \in$ $\boldsymbol{U}_{h}$ be two discrete functions and denote by $\mathbb{W}_{h}=\left(\mathbb{W}_{h, i}\right)_{1 \leqslant i \leqslant d} \in \mathbb{U}_{h}$ and $\nabla_{h}=$ $\left(\nabla_{h, i}\right)_{1 \leqslant i \leqslant d} \in \mathbb{U}_{h}$ the corresponding vectors of DOFs, where, for all $i \in\{1, \ldots, d\}$, 


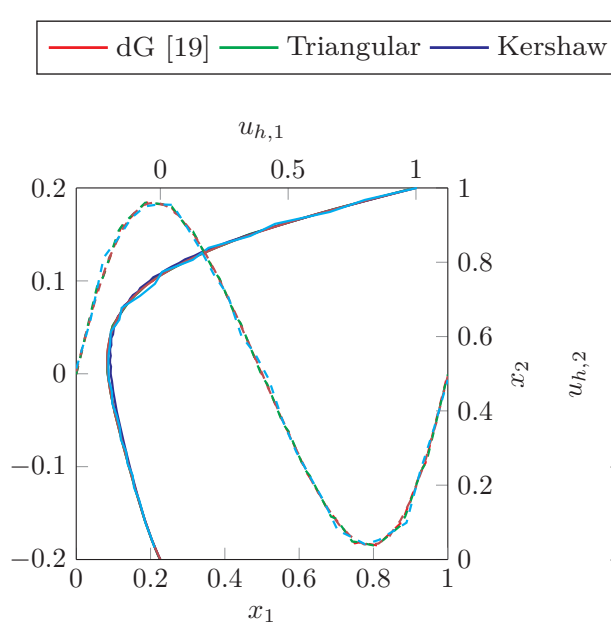

(A) Coarse meshes

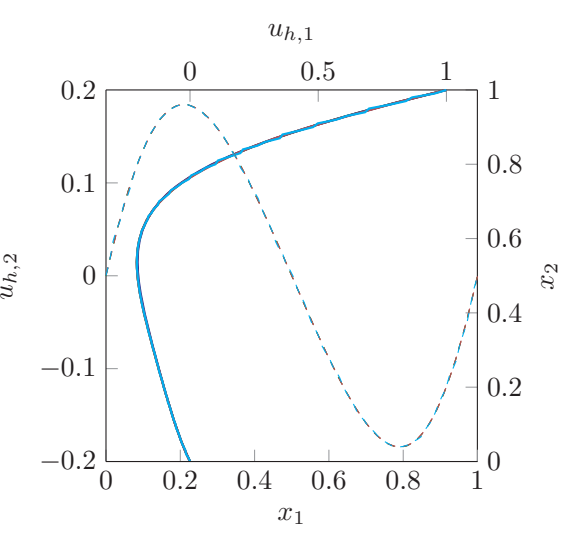

(B) Fine meshes

FiguRE 4. Results for the closed cavity problem on a coarse and a fine mesh extracted from the mesh families of Figure 3 Solid lines: horizontal displacement $u_{h, 1}$ along the vertical centerline. Dashed lines: vertical displacement $u_{h, 2}$ along the horizontal centerline.

$\mathbb{W}_{h, i}$ and $\nabla_{h, i}$ are the vectors of DOFs associated to the $i$ th components of $\boldsymbol{w}_{h}$ and $\boldsymbol{v}_{h}$ respectively. Our goal is to show that there exists a family of numerical fluxes $\left(\boldsymbol{\Phi}_{K, F}\left(\mathbb{W}_{h}\right)\right)_{K \in \mathcal{K}_{h}, F \in \mathcal{F}_{K}}$ with $\boldsymbol{\Phi}_{K, F}\left(\mathbb{W}_{h}\right)=\left(\Phi_{K, F, i}\left(\mathbb{W}_{h}\right)\right)_{1 \leqslant i \leqslant d}$ such that

$$
a_{h}\left(\boldsymbol{w}_{h}, \boldsymbol{v}_{h}\right)=\sum_{i=1}^{d} \sum_{K \in \mathcal{K}_{h}} \sum_{F \in \mathcal{F}_{K}} \Phi_{K, F, i}\left(\mathbb{W}_{h}\right)\left(v_{F, i}-v_{K, i}\right),
$$

with $a_{h}$ defined by (32). The main interest of this alternative formulation is that it allows us to prove a local conservation property similar to those encountered in standard finite volume methods. Recalling the expression (40) for the bilinear form $a_{h}$ and using the cell center as a quadrature node to approximate the righthand side in each element, the discrete problem (31) in algebraic form reads: Find $\mathbb{u}_{h} \in \mathbb{U}_{h}$ such that for all $\nabla_{h} \in \mathbb{U}_{h}$ there holds,

$$
\sum_{i=1}^{d} \sum_{K \in \mathcal{K}_{h}} \sum_{F \in \mathcal{F}_{K}} \Phi_{K, F, i}\left(\boldsymbol{u}_{h}\right)\left(v_{F, i}-v_{K, i}\right)=\sum_{i=1}^{d} \sum_{K \in \mathcal{K}_{h}}|K| f_{i}\left(\boldsymbol{x}_{K}\right) v_{K, i} .
$$

Consider now an interface $F \in \mathcal{F}_{\mathcal{K}_{h}}^{\mathrm{i}}$ such that $F \subset \partial K_{1} \cap \partial K_{2}$, and let $\nabla_{h, i}$ be such that $v_{F, i}=1, v_{F^{\prime}, i}=0$ for all $F^{\prime} \in \mathcal{F}_{\mathcal{K}_{h}} \backslash\{F\}$, and $v_{K, i}=0$ for all $K \in \mathcal{K}_{h}$. There follows from (41),

$$
\Phi_{K_{1}, F, i}\left(u_{h}\right)=-\Phi_{K_{2}, F, i}\left(u_{h}\right),
$$

i.e., the method is locally conservative. An important remark is that the loading term does not appear in (42) since its approximation in (41) only involves cell DOFs. 
Proposition 22 (Flux formulation). For all $\boldsymbol{w}_{h}, \boldsymbol{v}_{h} \in \boldsymbol{U}_{h}$, the flux formulation (40) is obtained setting for all $K \in \mathcal{K}_{h}, F \in \mathcal{F}_{K}$, and $i \in\{1, \ldots, d\}$,

$$
\begin{aligned}
\Phi_{K, F, i}\left(\mathbb{W}_{h}\right):=\sum_{F^{\prime} \in \mathcal{F}_{K}}\left|K_{F^{\prime}}\right| & {\left[\mu \boldsymbol{G}_{K_{F^{\prime}}}\left(\mathbb{W}_{h, i}\right) \cdot \boldsymbol{y}_{F^{\prime}, F}^{K}\right.} \\
& \left.+\left(\sum_{j=1}^{d} \mu \boldsymbol{G}_{K_{F^{\prime}}}\left(\mathbb{W}_{h, j}\right)+\lambda \boldsymbol{G}_{K}\left(\mathbb{W}_{h, j}\right)\right) \cdot \boldsymbol{e}_{j}\left(\boldsymbol{y}_{F^{\prime}, F}^{K} \cdot \boldsymbol{e}_{i}\right)\right],
\end{aligned}
$$

where $\mathbb{w}_{h}, \nabla_{h} \in \mathbb{U}_{h}$ are the vectors of DOFs associated to $\boldsymbol{w}_{h}$ and $\boldsymbol{v}_{h}$, respectively, $\left(\boldsymbol{e}_{i}\right)_{1 \leqslant i \leqslant d}$ denotes the canonical basis of $\mathbb{R}^{d}$, and

$$
\boldsymbol{y}_{F^{\prime}, F}^{K}:= \begin{cases}\frac{|F|}{|K|} \boldsymbol{n}_{K, F}+\frac{\eta}{d_{K, F}}\left(1-\frac{|F|}{|K|} \boldsymbol{n}_{K, F} \cdot\left(\overline{\boldsymbol{x}}_{F}-\boldsymbol{x}_{K}\right)\right) \boldsymbol{n}_{K, F} & \text { if } F=F^{\prime} \\ \frac{|F|}{|K|} \boldsymbol{n}_{K, F}-\frac{\eta}{d_{K, F^{\prime}}|K|}|F| \boldsymbol{n}_{K, F} \cdot\left(\overline{\boldsymbol{x}}_{F^{\prime}}-\boldsymbol{x}_{K}\right) \boldsymbol{n}_{K, F^{\prime}} & \text { otherwise. }\end{cases}
$$

Proof. For all $\nabla_{h} \in \mathbb{V}_{h}$, all $K \in \mathcal{K}_{h}$, and all $F^{\prime} \in \mathcal{F}_{K}$ there holds with $\boldsymbol{G}_{K_{F^{\prime}}}\left(\nabla_{h}\right)$ defined by (8) (cf. [25, eq. (26) et seq.]),

$$
\boldsymbol{G}_{K_{F^{\prime}}}\left(\nabla_{h}\right)=\sum_{F \in \mathcal{F}_{K}} \boldsymbol{y}_{F^{\prime}, F}^{K}\left(v_{F}-v_{K}\right) \text {. }
$$

Using (8) and (10), and observing that

$$
\left(\lambda D_{h}\left(\boldsymbol{w}_{h}\right), D_{h}\left(\boldsymbol{v}_{h}\right)\right)_{L^{2}(\Omega)}=\left(\lambda D_{h}\left(\boldsymbol{w}_{h}\right), \boldsymbol{\nabla}_{h} \cdot \boldsymbol{v}_{h}\right)_{L^{2}(\Omega)}
$$

owing to (20) together with the properties of the $L^{2}$-orthogonal projector, it is inferred that

$$
\begin{aligned}
a_{h}\left(\boldsymbol{w}_{h}, \boldsymbol{v}_{h}\right)=\sum_{i=1}^{d} \sum_{K \in \mathcal{K}_{h}} \sum_{F^{\prime} \in \mathcal{F}_{K}}\left|K_{F^{\prime}}\right|\left[\mu \boldsymbol{G}_{K_{F^{\prime}}}\left(\mathbb{W}_{h, i}\right)\right. \\
\left.\quad+\left(\sum_{j=1}^{d} \mu \boldsymbol{G}_{K_{F^{\prime}}}\left(\mathbb{W}_{h, j}\right) \cdot \boldsymbol{e}_{j}+\lambda \boldsymbol{G}_{K}\left(\mathbb{W}_{h, j}\right) \cdot \boldsymbol{e}_{j}\right) \boldsymbol{e}_{i}\right] \cdot \boldsymbol{G}_{K_{F^{\prime}}}\left(\mathbb{v}_{h, i}\right) .
\end{aligned}
$$

The conclusion follows using the expression (44) for $\boldsymbol{G}_{K_{F^{\prime}}}\left(\nabla_{h, i}\right)$ and exchanging the sums of indices $F$ and $F^{\prime}$.

Link with the Crouzeix-Raviart solution on matching simplicial meshes. Assume that $\mathcal{K}_{h}$ is a matching simplicial mesh and let

$$
\mathbb{C R}_{0}\left(\mathcal{K}_{h}\right):=\left\{v_{h} \in \mathbb{C R}\left(\mathcal{K}_{h}\right) \mid v_{h}\left(\overline{\boldsymbol{x}}_{F}\right)=0, \forall F \in \mathcal{F}_{\mathcal{K}_{h}}^{\mathrm{b}}\right\},
$$

and $\hat{\boldsymbol{U}}_{h}:=\mathbb{C R}_{0}\left(\mathcal{K}_{h}\right)^{d}$. We show in this section that a suitable modification of the right-hand side allows us to recover the Crouzeix-Raviart solution $\hat{\boldsymbol{u}}_{h} \in \hat{\boldsymbol{U}}_{h}$ such that (cf. [9]),

$$
a_{h}\left(\hat{\boldsymbol{u}}_{h}, \boldsymbol{v}_{h}\right)=\left(\boldsymbol{f}, \boldsymbol{v}_{h}\right)_{L^{2}(\Omega)^{d}} \quad \forall \boldsymbol{v}_{h} \in \hat{\boldsymbol{U}}_{h} .
$$

Let $W\left(\mathcal{P}_{h}\right):=\left\{v \in H^{1}\left(\mathcal{P}_{h}\right) \mid\langle\llbracket v \rrbracket\rangle_{F}=0\right.$ for all $\left.F \in \mathcal{F}_{\mathcal{P}_{h}}^{\mathrm{i}}\right\}$, and denote by $\mathcal{I}_{h}^{\mathbb{C R}}$ : $W\left(\mathcal{P}_{h}\right) \rightarrow \mathbb{C R}\left(\mathcal{K}_{h}\right)$ the interpolator that maps a function $v \in W\left(\mathcal{P}_{h}\right)$ on the function $v_{h}:=\mathcal{I}_{h}^{\mathbb{C R}}(v) \in \mathbb{C R}\left(\mathcal{K}_{h}\right)$ such that $v_{h}\left(\overline{\boldsymbol{x}}_{F}\right)=\langle v\rangle_{F}$ for all $F \in \mathcal{F}_{\mathcal{K}_{h}}$. We consider the following variation of (31): Find $\boldsymbol{u}_{h} \in \boldsymbol{U}_{h}$ such that

$$
a_{h}\left(\boldsymbol{u}_{h}, \boldsymbol{v}_{h}\right)=\left(\boldsymbol{f}, \mathcal{I}_{h}^{\mathbb{C R}}\left(\boldsymbol{v}_{h}\right)\right)_{L^{2}(\Omega)^{d}} \quad \forall \boldsymbol{v}_{h} \in \boldsymbol{U}_{h},
$$

where the sole difference with respect to (31) lies in the treatment of the right-hand side. 
Lemma 23 (Relation between (45) and (46)). There holds $\boldsymbol{u}_{h}=\hat{\boldsymbol{u}}_{h}$.

Proof. Let $\mathbb{U}_{h} \ni \hat{\mathrm{u}}_{h}=\left(\hat{\mathbb{U}}_{h, i}\right)_{1 \leqslant i \leqslant d}$ be such that, for $i \in\{1, \ldots, d\}$,

$$
\hat{\mathrm{U}}_{h, i}=\left(\left(\hat{u}_{h, i}\left(\boldsymbol{x}_{K}\right)\right)_{K \in \mathcal{K}_{h}},\left(\hat{u}_{h, i}\left(\overline{\boldsymbol{x}}_{F}\right)\right)_{F \in \mathcal{F}_{\mathcal{K}_{h}}}\right) \in \mathbb{V}_{h, 0} \text {. }
$$

Clearly, for all $K \in \mathcal{K}_{h}$, all $F \in \mathcal{F}_{K}$, and all $i \in\{1, \ldots, d\}, \boldsymbol{R}_{K_{F}}\left(\hat{\mathrm{u}}_{h, i}\right)=\mathbf{0}$, hence $\boldsymbol{G}_{K_{F}}\left(\hat{\mathrm{u}}_{h, i}\right)=\boldsymbol{G}_{K}\left(\hat{\mathrm{u}}_{h, i}\right)=\left(\boldsymbol{\nabla}_{h} \hat{u}_{h, i}\right)_{\mid K}$. As a consequence, $\hat{\boldsymbol{u}}_{h}=\mathfrak{R}_{h}\left(\hat{\mathrm{u}}_{h}\right)$. Accounting for this fact, there holds for all $\boldsymbol{v}_{h} \in \boldsymbol{U}_{h}$ such that $\boldsymbol{v}_{h}=\mathfrak{R}_{h}\left(\nabla_{h}\right)$ with $\nabla_{h} \in \mathbb{U}_{h}$,

$$
\begin{aligned}
a_{h}\left(\hat{\boldsymbol{u}}_{h}, \boldsymbol{v}_{h}\right)= & \sum_{i=1}^{d} \sum_{K \in \mathcal{K}_{h}} \sum_{F \in \mathcal{F}_{K}}\left|K_{F}\right|\left\{\mu \boldsymbol{G}_{K}\left(\hat{\mathrm{u}}_{h, i}\right) \cdot \boldsymbol{G}_{K_{F}}\left(\mathbb{\nabla}_{h, i}\right)+\mu \boldsymbol{G}_{K}\left(\hat{\mathrm{u}}_{h, i}\right) \cdot \boldsymbol{e}_{i} D_{K_{F}}\left(\mathbb{\nabla}_{h}\right)\right. \\
& \left.+\lambda \boldsymbol{G}_{K}\left(\hat{\mathrm{u}}_{h, i}\right) \cdot \boldsymbol{e}_{i} D_{K}\left(\mathbb{v}_{h}\right)\right\} \\
= & \sum_{i=1}^{d} \sum_{K \in \mathcal{K}_{h}}|K|\left\{\mu \boldsymbol{G}_{K}\left(\hat{\mathrm{u}}_{h, i}\right) \cdot \boldsymbol{G}_{K}\left(\nabla_{h, i}\right)+(\mu+\lambda) \boldsymbol{G}_{K}\left(\hat{\mathrm{u}}_{h, i}\right) \cdot \boldsymbol{e}_{i} D_{K}\left(\nabla_{h}\right)\right\} \\
= & a_{h}\left(\hat{\boldsymbol{u}}_{h}, \mathcal{I}_{h}^{\mathbb{C R}}\left(\boldsymbol{v}_{h}\right)\right)=\left(\boldsymbol{f}, \mathcal{I}_{h}^{\mathbb{C R}}\left(\boldsymbol{v}_{h}\right)\right)_{L^{2}(\Omega)^{d}},
\end{aligned}
$$

where the first passage is a consequence of (17) and we have let, for the sake of conciseness $D_{K}\left(\nabla_{h}\right):=\sum_{j=1}^{d} \boldsymbol{G}_{K}\left(\nabla_{h, j}\right) \cdot \boldsymbol{e}_{j}$ and $D_{K_{F}}\left(\nabla_{h}\right):=\sum_{j=1}^{d} \boldsymbol{G}_{K_{F}}\left(\nabla_{h, j}\right) \cdot \boldsymbol{e}_{j}$. Owing to the coercivity of $a_{h}$, problem (46) admits a unique solution and we therefore conclude that $\hat{\boldsymbol{u}}_{h}=\boldsymbol{u}_{h}$.

4.2.5. Mixed-type boundary conditions. We describe here a possible modification of the method that allows us to account for homogeneous mixed-type boundary conditions (the generalization to the nonhomogeneous case is straightforward). The continuous problem reads

$$
\begin{array}{rlrl}
-\boldsymbol{\nabla} \cdot \underline{\underline{\sigma}}(\boldsymbol{u}) & =\boldsymbol{f} & & \text { in } \Omega, \\
\boldsymbol{u} & =\mathbf{0} & & \text { on } \partial \Omega^{\mathrm{D}}, \\
\underline{\underline{\sigma}}(\boldsymbol{u}) \boldsymbol{n}=\mathbf{0} & & \text { on } \partial \Omega^{\mathrm{N}},
\end{array}
$$

where $\partial \Omega^{\mathrm{D}}$ and $\partial \Omega^{\mathrm{N}}$ are such that $\partial \Omega^{\mathrm{D}} \neq \varnothing, \partial \Omega^{\mathrm{D}} \cap \partial \Omega^{\mathrm{N}}=\varnothing$, and $\partial \Omega^{\mathrm{D}} \cup \partial \Omega^{\mathrm{N}}=\partial \Omega$. Let $\boldsymbol{U}:=H_{\mathrm{D}}^{1}(\Omega)^{d}$ with $H_{\mathrm{D}}^{1}(\Omega):=\left\{v \in H^{1}(\Omega) \mid v_{\mid \partial \Omega^{\mathrm{D}}}=0\right\}$. In this case, the weak formulation (28) is no longer equivalent to the continuous problem (47), and (26) must be used instead: Find $\boldsymbol{u} \in \boldsymbol{U}$ such that

$$
\tilde{a}(\boldsymbol{u}, \boldsymbol{v})=(\boldsymbol{f}, \boldsymbol{v})_{L^{2}(\Omega)^{d}} \quad \forall \boldsymbol{v} \in \boldsymbol{U} .
$$

The well-posedness of the weak formulation (48) relies on Korn's inequality (27), which is still valid on the space $H_{\mathrm{D}}^{1}(\Omega)^{d}$ (up to a multiplicative constant depending on $\partial \Omega^{\mathrm{D}}$ ). Likewise, the regularity estimate of Lemma 14 holds for problem (47) in the case of a convex polygonal domain with the above definition of $\boldsymbol{U}$ and $\boldsymbol{U}_{*}:=$ $\boldsymbol{U} \cap H^{2}(\Omega)^{d}$. We introduce the following $H_{\mathrm{D}}^{1}$-like discrete space to approximate the displacement field:

$$
\mathfrak{C} \mathfrak{R}_{\mathrm{D}}\left(\mathcal{K}_{h}\right):=\mathfrak{R}_{h}\left(\mathbb{V}_{h, \mathrm{D}}\right), \quad \mathbb{V}_{h, \mathrm{D}}=\left\{\mathbb{}_{h} \in \mathbb{V}_{h} \mid v_{F}=0, \forall F \in \mathcal{F}_{\mathcal{K}_{h}^{\mathrm{b}, \mathrm{D}}}\right\},
$$

where $\mathcal{F}_{\mathcal{K}_{h}}^{\mathrm{b}, \mathrm{D}}:=\left\{F \in \mathcal{F}_{\mathcal{K}_{h}}^{\mathrm{b}} \mid F \subset \partial \Omega^{\mathrm{D}}\right\}$. We also introduce the set $\mathcal{F}_{\mathcal{K}_{h}^{\mathrm{N}}}^{\mathrm{b}}:=$ $\mathcal{F}_{\mathcal{K}_{h}}^{\mathrm{b}} \backslash \mathcal{F}_{\mathcal{K}_{h}}^{\text {b,D }}$. We first note that $\left\|\nabla_{h} v_{h}\right\|_{L^{2}(\Omega)^{d}}$ is still a norm on $\mathfrak{C} \Re_{\mathrm{D}}\left(\mathcal{K}_{h}\right)$. Owing 
to the nonconformity of the space we consider, Korn's inequality only holds in the following generalized sense (cf. [7, eq. (1.12)]):

$$
\left\|\nabla_{h} \boldsymbol{v}\right\|_{L^{2}(\Omega)^{d, d}} \leqslant C_{\mathrm{K}}\left(\left\|\underline{\underline{\varepsilon_{h}}}(\boldsymbol{v})\right\|_{L^{2}(\Omega)^{d, d}}^{2}+|\boldsymbol{v}|_{\mathrm{J}, \mathrm{D}}^{2}\right)^{\frac{1}{2}}
$$

for any $\boldsymbol{v}=\left(v_{i}\right)_{1 \leqslant i \leqslant d} \in H^{1}\left(\mathcal{P}_{h}\right)^{d}$, where $C_{\mathrm{K}}>0$ is independent of the meshsize (but depends on $\Omega$ and on the mesh regularity parameters), and $|\boldsymbol{v}|_{\mathrm{J}, \mathrm{D}}^{2}:=\sum_{i=1}^{d}\left|v_{i}\right|_{\mathrm{J}, \mathrm{D}}^{2}$

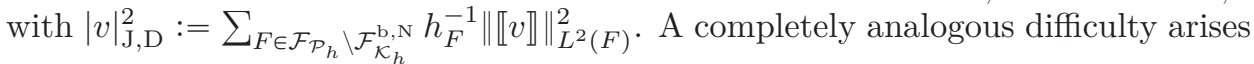
for the standard Crouzeix-Raviart space. To overcome this problem, we take inspiration from [29] and consider a discrete version of the bilinear form $\tilde{a}$ which incorporates a least-square penalization of jumps. More specifically, letting $\boldsymbol{U}_{h}:=$ $\mathfrak{C} \mathfrak{R}_{\mathrm{D}}\left(\mathcal{K}_{h}\right)^{d}$, the discrete problem reads: Find $\boldsymbol{u}_{h} \in \boldsymbol{U}_{h}$ such that

$$
\tilde{a}_{h}\left(\boldsymbol{u}_{h}, \boldsymbol{v}_{h}\right)=\left(\boldsymbol{f}, \boldsymbol{v}_{h}\right)_{L^{2}(\Omega)^{d}} \quad \forall \boldsymbol{v}_{h} \in \boldsymbol{U}_{h},
$$

where the discrete bilinear form $\tilde{a}_{h}$ is given by

$$
\begin{aligned}
\tilde{a}_{h}(\boldsymbol{w}, \boldsymbol{v}):= & \left(2 \mu \underline{\underline{\varepsilon_{h}}}(\boldsymbol{w}), \underline{\underline{\varepsilon_{h}}}(\boldsymbol{v})\right)_{L^{2}(\Omega)^{d, d}}+\left(\lambda D_{h}(\boldsymbol{w}), D_{h}(\boldsymbol{v})\right)_{L^{2}(\Omega)} \\
& +\sum_{F \in \mathcal{F}_{\mathcal{P}_{h} \backslash \mathcal{F}_{\mathcal{K}_{h}}^{\mathrm{b}, \mathrm{N}}}} h_{F}^{-1}(2 \mu \llbracket \boldsymbol{w} \rrbracket, \llbracket \boldsymbol{v} \rrbracket)_{L^{2}(F)^{d}} .
\end{aligned}
$$

It is worth observing that, while the idea of penalizing jumps to recover a discrete Korn's inequality appears natural in the present approach, this is not the case in other related frameworks for which the notion of affine reconstruction does not necessarily make sense.

Remark 24 (Implementation). We stress that in this case it is not possible to integrate the penalty term in (50) using the face barycenter as a quadrature point, since this would yield a vanishing contribution. A quadrature rule exact for polynomials of degree at least 2 must be used instead. We shall also stress that it is possible to reformulate the method in terms of face unknowns only as for the pure displacement problem (cf. Remark 15), since, actually, the penalty term does not establish any link between neighboring cell unknowns. However, note that this jump's penalization highly increases the stencil of the method in terms of connections between face unknowns.

The energy norm associated to the bilinear form $\tilde{a}_{h}$ is

$$
\|\boldsymbol{v}\|_{\mathrm{el}}^{2}:=\tilde{a}_{h}(\boldsymbol{v}, \boldsymbol{v})=\left\|(2 \mu)^{1 / 2} \underline{\underline{\varepsilon_{h}}}(\boldsymbol{v})\right\|_{L^{2}(\Omega)^{d, d}}^{2}+\left\|\lambda^{1 / 2} D_{h}(\boldsymbol{v})\right\|_{L^{2}(\Omega)}^{2}+\left|(2 \mu)^{1 / 2} \boldsymbol{v}\right|_{\mathrm{J}, \mathrm{D}}^{2} .
$$

Lemma 25 (Coercivity). There holds for all $\boldsymbol{v}_{h} \in \boldsymbol{U}_{h}$,

$$
\tilde{a}_{h}\left(\boldsymbol{v}_{h}, \boldsymbol{v}_{h}\right)=\left\|\boldsymbol{v}_{h}\right\|_{\mathrm{el}}^{2} \geqslant 2 \mu C_{\mathrm{K}}^{-2}\left\|\nabla_{h} \boldsymbol{v}_{h}\right\|_{L^{2}(\Omega)^{d, d}}^{2} .
$$

The well-posedness of problem (49) is now straightforward. Defining $\boldsymbol{U}_{* h}:=$ $\boldsymbol{U}_{*}+\boldsymbol{U}_{h}$ and extending $\tilde{a}_{h}$ to $\boldsymbol{U}_{* h} \times \boldsymbol{U}_{* h}$, the convergence analysis can be carried out in a similar way as in Section 4.2.2 Assuming $\boldsymbol{u} \in \boldsymbol{U}_{*}$, the consistency error becomes, for all $\boldsymbol{v}_{h} \in \boldsymbol{U}_{h}$,

$$
\mathcal{E}_{h}\left(\boldsymbol{v}_{h}\right):=\sum_{F \in \mathcal{F}_{\mathcal{P}_{h}} \backslash \mathcal{F}_{\mathcal{K}_{h}}^{\mathrm{b}, \mathrm{N}}}\left(\underline{\underline{\sigma}}(\boldsymbol{u}) \boldsymbol{n}_{F}, \llbracket \boldsymbol{v}_{h} \rrbracket\right)_{L^{2}(F)^{d}}+\left(\lambda\left(D_{h}(\boldsymbol{u})-\boldsymbol{\nabla} \cdot \boldsymbol{u}\right), \nabla_{h} \cdot \boldsymbol{v}_{h}\right)_{L^{2}(\Omega)}
$$


Using the same notation as in the proof of Theorem [18 with $\boldsymbol{w}_{h}:=\mathcal{I}_{h}^{\mathfrak{C} R}(\boldsymbol{u}) \in \boldsymbol{U}_{h}$, the term $\mathfrak{T}_{1}$ can be handled as

$$
\mathfrak{T}_{1} \leqslant\left\|\boldsymbol{u}-\boldsymbol{w}_{h}\right\|_{\mathrm{el}} \lesssim h\|\boldsymbol{u}\|_{H^{2}(\Omega)^{d}}+h\left|\lambda^{1 / 2} \nabla \cdot \boldsymbol{u}\right|_{H^{1}(\Omega)},
$$

using Lemma 10, Corollary [12, and the trace inequality (6) with $\mathcal{S}_{h}=\mathcal{P}_{h}$ combined with Lemma 10] respectively, to treat the three terms in the right-hand side of (51) with $\boldsymbol{v}=\boldsymbol{u}-\boldsymbol{w}_{h}$. The remaining terms can be treated by using exactly the same arguments as in the proof of Theorem 18, As a result, if $d=2$, we again obtain a locking-free error estimate of the form (30).

4.3. Stokes: inf-sup stability and large irrotational body forces. In this section we briefly discuss an inf-sup stable method for the Stokes problem with velocity components in $\mathfrak{C} \mathfrak{R}\left(\mathcal{K}_{h}\right)$ and piecewise constant pressure. We also pinpoint a general strategy for the treatment of large irrotational forces and apply it to the proposed method.

4.3.1. Setting and discretization. The link between locking-free approximations of linear elasticity and inf-sup stable approximations of the Stokes problem is wellknown; cf., e.g., the discussion in [10, Section VI.3]. We consider here a Newtonian fluid of constant unit viscosity whose motion is governed by the Stokes equations,

$$
\begin{array}{rlrl}
-\triangle \boldsymbol{u}+\nabla p & =\boldsymbol{f} & & \text { in } \Omega, \\
\nabla \cdot \boldsymbol{u} & =0 & & \text { in } \Omega, \\
\boldsymbol{u} & =\mathbf{0} & & \text { on } \partial \Omega, \\
\int_{\Omega} p & =0, &
\end{array}
$$

where $f \in L^{2}(\Omega)^{d}$ represents the volumetric body force acting on the fluid. Let $\boldsymbol{U}:=H_{0}^{1}(\Omega)^{d}$, and $P:=L_{0}^{2}(\Omega)$ with $L_{0}^{2}(\Omega):=\left\{q \in L^{2}(\Omega) \mid \int_{\Omega} q=0\right\}$. The weak formulation of problem (52) reads: Find $(\boldsymbol{u}, p) \in \boldsymbol{U} \times P$ such that

$$
a(\boldsymbol{u}, \boldsymbol{v})+b(\boldsymbol{v}, p)-b(\boldsymbol{u}, q)=(\boldsymbol{f}, \boldsymbol{v})_{L^{2}(\Omega)^{d}} \quad \forall(\boldsymbol{v}, q) \in \boldsymbol{U} \times P,
$$

where $a(\boldsymbol{u}, \boldsymbol{v}):=(\boldsymbol{\nabla} \boldsymbol{u}, \boldsymbol{\nabla v})_{L^{2}(\Omega)^{d, d}}$ and $b(\boldsymbol{v}, p):=-(\boldsymbol{\nabla} \cdot \boldsymbol{v}, p)_{L^{2}(\Omega)}$. To approximate (53), let $\mathcal{K}_{h}$ belong to an admissible mesh sequence and define the following discrete spaces:

$$
\boldsymbol{U}_{h}:=\mathfrak{C} \mathfrak{R}_{0}\left(\mathcal{K}_{h}\right)^{d}, \quad P_{h}:=\mathbb{P}_{d}^{0}\left(\mathcal{K}_{h}\right) \cap L_{0}^{2}(\Omega) .
$$

We equip $\boldsymbol{U}_{h}$ with the norm $\|\boldsymbol{v}\|_{\boldsymbol{U}}:=\left\|\boldsymbol{\nabla}_{h} \boldsymbol{v}\right\|_{L^{2}(\Omega)^{d, d}}$ (see Proposition 13), and $P_{h}$ with the norm $\|q\|_{P}:=\|q\|_{L^{2}(\Omega)}$. We assume in the following $\eta=d$ in (9), so that the continuity of mean values stated in Lemma 8 holds, and consider the following discrete problem: Find $\left(\boldsymbol{u}_{h}, p_{h}\right) \in \boldsymbol{U}_{h} \times P_{h}$ such that

$$
a_{h}\left(\boldsymbol{u}_{h}, \boldsymbol{v}_{h}\right)+b_{h}\left(\boldsymbol{v}_{h}, p_{h}\right)-b_{h}\left(\boldsymbol{u}_{h}, q_{h}\right)=\left(\boldsymbol{f}, \boldsymbol{v}_{h}\right)_{L^{2}(\Omega)^{d}} \quad \forall\left(\boldsymbol{v}_{h}, q_{h}\right) \in \boldsymbol{U}_{h} \times P_{h},
$$

where $a_{h}(\boldsymbol{w}, \boldsymbol{v}):=\left(\boldsymbol{\nabla}_{h} \boldsymbol{w}, \boldsymbol{\nabla}_{h} \boldsymbol{v}\right)_{L^{2}(\Omega)^{d, d}}$ and $b_{h}(\boldsymbol{v}, q):=-\left(\boldsymbol{\nabla}_{h} \cdot \boldsymbol{v}, q\right)_{L^{2}(\Omega)}$. For all $\left(\boldsymbol{v}_{h}, q_{h}\right) \in \boldsymbol{U}_{h} \times P_{h}$ there holds

$$
b_{h}\left(\boldsymbol{v}_{h}, q_{h}\right)=-\left(D_{h}\left(\boldsymbol{v}_{h}\right), q_{h}\right)_{L^{2}(\Omega)} .
$$


Lemma 26 (inf-sup stability for $b_{h}$ ). There exists $\beta>0$ independent of the meshsize, such that, for all $q_{h} \in P_{h}$,

$$
\beta\left\|q_{h}\right\|_{P} \leqslant \sup _{\boldsymbol{w}_{h} \in \boldsymbol{U}_{h} \backslash\{\mathbf{0}\}} \frac{b_{h}\left(\boldsymbol{w}_{h}, q_{h}\right)}{\left\|\boldsymbol{w}_{h}\right\|_{\boldsymbol{U}}} .
$$

Proof. We sketch the proof for the sake of completeness. Let $\boldsymbol{v} \in \boldsymbol{U}$ and $\boldsymbol{v}_{h}:=$ $\mathcal{I}_{h}^{\mathfrak{C} R}(\boldsymbol{v}) \in \boldsymbol{U}_{h}$. Using the triangular inequality followed by (16) with $l=0$, it is inferred with $C$ independent of the meshsize, $\left\|\boldsymbol{v}_{h}\right\|_{\boldsymbol{U}} \leqslant\left\|\boldsymbol{\nabla} \boldsymbol{v}-\boldsymbol{\nabla}_{h} \boldsymbol{v}_{h}\right\|_{L^{2}(\Omega)^{d, d}}+$ $\|\boldsymbol{\nabla} \boldsymbol{v}\|_{L^{2}(\Omega)^{d, d}} \leqslant C\|\boldsymbol{v}\|_{H^{1}(\Omega)^{d}}$. This $H^{1}$-stability property together with Corollary 12 and (55) show that $\mathcal{I}_{h}^{\mathfrak{C} \Re}$ can play the role of a Fortin operator, and (56) results from an application of [10, Proposition II.8].

The well-posedness of the discrete problem (54) follows from Lemma 26 together with the coercivity of $a_{h}$ (an immediate consequence of Proposition 13). Moreover, as pointed out in [10, Section IV.3], the inf-sup condition proved in Lemma26] is intimately linked to the good behavior of the method (31) in the quasi-incompressible limit. In fact, for inf-sup stable approximations of the Stokes problem with discontinuous pressures, one can obtain a locking-free primal method for the pure displacement problem of elasticity by performing static condensation of pressures, which is equivalent to introducing a projection on divergence terms (this strategy can, e.g., be pursued for the method of [5, 6]).

Using classical arguments, one can prove the following result, which is invoked in the discussion of Section 4.3.3. As in Theorem 18, the continuity of mean values at interfaces is used to bound the consistency error. Optimal error estimates for the $L^{2}$-error on the velocity can also be derived using the Aubin-Nitsche trick.

Theorem 27 (Error estimate for (54)). Assume $\boldsymbol{u} \in \boldsymbol{U} \cap H^{2}(\Omega)^{d}$ and $p \in P \cap$ $H^{1}(\Omega)$. Then, there holds with $C>0$ independent of the meshsize, of $\boldsymbol{u}$, and of $p$,

$$
\left\|\left(\boldsymbol{u}-\boldsymbol{u}_{h}, p-p_{h}\right)\right\|_{\text {sto }} \leqslant C h \mathcal{N}_{\text {sto }}(\boldsymbol{u}, p),
$$

where

$$
\|(\boldsymbol{v}, q)\|_{\text {sto }}^{2}:=\|\boldsymbol{v}\|_{\boldsymbol{U}}^{2}+\|q\|_{P}^{2} \quad \text { and } \quad \mathcal{N}_{\text {sto }}(\boldsymbol{u}, p):=\|\boldsymbol{u}\|_{H^{2}(\Omega)^{d}}+\|p\|_{H^{1}(\Omega)} .
$$

4.3.2. Variations and links with finite volume and finite element methods.

Flux formulation and local conservation. Let $\left(\boldsymbol{w}_{h}, r_{h}\right),\left(\boldsymbol{v}_{h}, q_{h}\right) \in \boldsymbol{U}_{h} \times P_{h}$ be two discrete functions, and denote by $\left(\mathbb{W}_{h}, \mathbb{v}_{h}\right),\left(\mathbb{v}_{h}, \mathbb{q}_{h}\right) \in \mathbb{U}_{h} \times \mathbb{P}_{h}$ the corresponding vectors of DOFs, where we have set $\mathbb{U}_{h}:=\mathbb{V}_{h, 0}^{d}$ and $\mathbb{P}_{h}:=\left\{\mathbb{q}_{h} \in\right.$ $\left.\mathbb{R}^{\mathcal{K}_{h}}\left|\sum_{K \in \mathcal{K}_{h}}\right| K \mid q_{K}=0\right\}$. Then, proceeding as in Section 4.2.4 one can show that for the two families of fluxes $\left(\boldsymbol{\Phi}_{K, F}\left(\mathbb{W}_{h}, \mathbb{r}_{h}\right)\right)_{K \in \mathcal{K}_{h}, F \in \mathcal{F}_{K}}$ with $\boldsymbol{\Phi}_{K, F}\left(\mathbb{W}_{h}, \mathbb{r}_{h}\right)=$ $\left(\Phi_{K, F, i}\left(\mathbb{W}_{h}, \mathbb{\varpi}_{h}\right)\right)_{1 \leqslant i \leqslant d}$ and $\left(\phi_{F}\left(\mathbb{W}_{h}\right)\right)_{F \in \mathcal{F}_{\mathcal{K}_{h}}}$ such that (the expression for the vectors $\boldsymbol{y}_{F^{\prime}, F}^{K}$ is provided in Proposition 22)

$$
\begin{aligned}
\Phi_{K, F, i}\left(\mathbb{W}_{h}, \mathbb{r}_{h}\right) & :=\sum_{F^{\prime} \in \mathcal{F}_{K}}\left|K_{F^{\prime}}\right|\left(\boldsymbol{G}_{K_{F^{\prime}}}\left(\mathbb{W}_{h, i}\right)+r_{K} \boldsymbol{e}_{i}\right) \cdot \boldsymbol{y}_{F^{\prime}, F}^{K}, \\
\phi_{F}\left(\mathbb{W}_{h}\right) & :=|F| \sum_{j=1}^{d} w_{F, j} n_{F, j},
\end{aligned}
$$


there holds,

$$
\begin{aligned}
a_{h}\left(\boldsymbol{w}_{h}, \boldsymbol{v}_{h}\right)+b_{h}\left(\boldsymbol{v}_{h}, r_{h}\right) & =\sum_{i=1}^{d} \sum_{K \in \mathcal{K}_{h}} \sum_{F \in \mathcal{F}_{K}} \Phi_{K, F, i}\left(\mathbb{W}_{h}, \mathfrak{\varpi}_{h}\right)\left(v_{F, i}-v_{K, i}\right), \\
-b_{h}\left(\boldsymbol{w}_{h}, q_{h}\right) & =\sum_{F \in \mathcal{F}_{\mathcal{K}_{h}}} \phi_{F}\left(\mathbb{W}_{h}\right) \llbracket \mathbb{q}_{h} \rrbracket_{F},
\end{aligned}
$$

where $a_{h}$ and $b_{h}$ are defined as in Section 4.3.1 and, with a slight abuse in notation, we have set for all $F \in \mathcal{F}_{\mathcal{K}_{h}}, \llbracket q_{h} \rrbracket_{F}:=\llbracket q_{h} \rrbracket_{F}$. Approximating the right-hand side as $\sum_{i=1}^{d} \sum_{K \in \mathcal{K}_{h}}|K| f_{i}\left(\boldsymbol{x}_{K}\right) v_{K, i}$ in (54), and proceeding as in Section 4.2.4 one can prove that for every interface $F \in \mathcal{F}_{\mathcal{K}_{h}}^{\mathrm{i}}$ such that $F \subset \partial K_{1} \cap \partial K_{2}$ there holds $\boldsymbol{\Phi}_{K_{1}, F}\left(\mathbb{u}_{h}, \mathbb{P}_{h}\right)=-\boldsymbol{\Phi}_{K_{2}, F}\left(\mathbb{U}_{h}, \mathbb{P}_{h}\right)$, where $\mathbb{u}_{h}, \mathbb{P}_{h}$ are such that $\boldsymbol{u}_{h}:=\mathfrak{R}_{h}\left(\mathbb{U}_{h}\right)$ and $p_{h}:=\mathfrak{R}_{h}\left(\mathrm{p}_{h}\right)$ with $\left(\boldsymbol{u}_{h}, p_{h}\right) \in \boldsymbol{U}_{h} \times P_{h}$ solution of (54). Moreover, the mass flux $\phi_{F}\left(\mathbb{u}_{h}\right)$ is single-valued, and therefore conservative.

Link with the Crouzeix-Raviart solution on matching simplicial meshes. Assume $\mathcal{K}_{h}$ matching simplicial. The classical Crouzeix-Raviart/ $\mathbb{P}_{d}^{0}$ method consists in finding $\left(\hat{\boldsymbol{u}}_{h}, \hat{p}_{h}\right) \in \hat{\boldsymbol{U}}_{h} \times P_{h}$ with $\hat{\boldsymbol{U}}_{h}:=\mathbb{C R}_{0}\left(\mathcal{K}_{h}\right)^{d}$ such that

$$
a_{h}\left(\hat{\boldsymbol{u}}_{h}, \boldsymbol{v}_{h}\right)+b_{h}\left(\boldsymbol{v}_{h}, \hat{p}_{h}\right)-b_{h}\left(\hat{\boldsymbol{u}}_{h}, q_{h}\right)=\left(\boldsymbol{f}, \boldsymbol{v}_{h}\right)_{L^{2}(\Omega)^{d}} \quad \forall\left(\boldsymbol{v}_{h}, q_{h}\right) \in \hat{\boldsymbol{U}}_{h} \times P_{h} .
$$

Then, proceeding as in Section 4.2.4 one can easily show that the solution to (58) can be recovered replacing the right-hand side of (54) by $\left(\boldsymbol{f}, \mathcal{I}_{h}^{\mathbb{C R}}\left(\boldsymbol{v}_{h}\right)\right)_{L^{2}(\Omega)^{d}}$.

4.3.3. Large irrotational body forces. We close this section by discussing a general modification applicable to any suitable discretization of the Stokes equations that allows a proper treatment of large irrotational body forces, and we apply it to the method (54). We assume here that the following Helmholtz decomposition of the volumetric body forces in (52) is available:

$$
f=\Psi-\nabla \varphi
$$

where $\boldsymbol{\Psi} \in \boldsymbol{H}_{0}(\operatorname{div} ; \Omega):=\{\boldsymbol{v} \in \boldsymbol{H}(\operatorname{div} ; \Omega) \mid \boldsymbol{\nabla} \cdot \boldsymbol{v}=0\}$ is a solenoidal vector field and $\varphi \in H^{1}(\Omega) \cap L_{0}^{2}(\Omega)$ is a scalar potential. It has to be noted that the decomposition (59) is not always easy to obtain. The weak formulation of problem (52) with right-hand side given by (59) reads: Find $(\boldsymbol{u}, p) \in \boldsymbol{U} \times P$ such that

$$
a(\boldsymbol{u}, \boldsymbol{v})+b(\boldsymbol{v}, p)-b(\boldsymbol{u}, q)=l(\boldsymbol{v}) \quad \forall(\boldsymbol{v}, q) \in \boldsymbol{U} \times P,
$$

with bilinear forms $a$ and $b$ defined as in Section 4.3.1 and $l(\boldsymbol{v}):=(\boldsymbol{\Psi}, \boldsymbol{v})_{L^{2}(\Omega)^{d}}-$ $b(\boldsymbol{v}, \varphi)$. Denoting by $\left(\boldsymbol{u}_{\Psi}, p_{\Psi}\right)$ the solution to (60) with $\varphi \equiv 0$ (no irrotational body forces), there holds

$$
\boldsymbol{u}=\boldsymbol{u}_{\Psi}, \quad p=p_{\Psi}-\varphi .
$$

As pointed out in [27, mimicking or approaching property (61) at the discrete level is a key ingredient to obtain an accurate approximation of the velocity field for large values of $\|\varphi\|_{H^{1}(\Omega)}$. We hence consider the following approximation to (60): Find $\left(\boldsymbol{u}_{h}, p_{h}\right) \in \boldsymbol{U}_{h} \times P_{h}$ such that

$$
a_{h}\left(\boldsymbol{u}_{h}, \boldsymbol{v}_{h}\right)+b_{h}\left(\boldsymbol{v}_{h}, p_{h}\right)-b_{h}\left(\boldsymbol{u}_{h}, q_{h}\right)=l_{h}\left(\boldsymbol{v}_{h}\right) \quad \forall\left(\boldsymbol{v}_{h}, q_{h}\right) \in \boldsymbol{U}_{h} \times P_{h},
$$

with bilinear forms $a_{h}$ and $b_{h}$ defined as in Section 4.3.1 and

$$
l_{h}\left(\boldsymbol{v}_{h}\right):=\left(\boldsymbol{\Psi}, \boldsymbol{v}_{h}\right)_{L^{2}(\Omega)^{d}}-b_{h}\left(\boldsymbol{v}_{h}, \Pi_{h}^{0} \varphi\right) .
$$


The sole difference with respect to (54) lies in the treatment of the source term, which is designed so that the following property holds true.

Proposition 28 (Discrete counterpart of property (61)). Denote by $\left(\boldsymbol{u}_{\boldsymbol{\Psi}, h}, p_{\boldsymbol{\Psi}, h}\right)$ the solution to problem (62) with $\varphi \equiv 0$. There holds

$$
\boldsymbol{u}_{h}=\boldsymbol{u}_{\boldsymbol{\Psi}, h}, \quad p_{h}=p_{\boldsymbol{\Psi}, h}-\Pi_{h}^{0} \varphi .
$$

The following result shows that the velocity approximation is not affected by the irrotational part of the source term.

Theorem 29 (Error estimate for (62) ). Assume $\boldsymbol{u} \in \boldsymbol{U} \cap H^{2}(\Omega)^{d}$ and $p \in P \cap$ $H^{1}(\Omega)$. Then, there holds with real numbers $C_{1}>0$ and $C_{2}>0$ independent of the meshsize, of $\boldsymbol{u}$, and of $p$, but depending on the mesh regularity parameters and on $\Omega$,

$\left\|\boldsymbol{u}-\boldsymbol{u}_{h}\right\|_{\boldsymbol{U}} \leqslant C_{1} h \mathcal{N}_{\text {sto }}\left(\boldsymbol{u}_{\boldsymbol{\Psi}}, p_{\boldsymbol{\Psi}}\right), \quad\left\|p-p_{h}\right\|_{P} \leqslant C_{2} h\left(\mathcal{N}_{\text {sto }}\left(\boldsymbol{u}_{\boldsymbol{\Psi}}, p_{\boldsymbol{\Psi}}\right)+\|\varphi\|_{H^{1}(\Omega)}\right)$.

Proof. Using Theorem 27for the approximate solution to problem (60) with $\varphi \equiv 0$, we infer $\left\|\boldsymbol{u}_{\boldsymbol{\Psi}}-\boldsymbol{u}_{\boldsymbol{\Psi}, h}\right\|_{\boldsymbol{U}}+\left\|p_{\boldsymbol{\Psi}}-p_{\boldsymbol{\Psi}, h}\right\|_{P} \leqslant C h \mathcal{N}_{\text {sto }}\left(\boldsymbol{u}_{\boldsymbol{\Psi}}, p_{\boldsymbol{\Psi}}\right)$, where $C>0$ has the same dependencies as $C_{1}$ and $C_{2}$. The estimate for $\left\|\boldsymbol{u}-\boldsymbol{u}_{h}\right\|_{\boldsymbol{U}}$ is an immediate consequence of (61) and Proposition 28 To estimate $\left\|p-p_{h}\right\|_{P}$ we invoke again (61) and Proposition 28 to infer $\left\|p-p_{h}\right\|_{P} \leqslant\left\|p_{\boldsymbol{\Psi}}-p_{\boldsymbol{\Psi}, h}\right\|_{P}+\left\|\varphi-\Pi_{h}^{0} \varphi\right\|_{P}$, and conclude using the above estimate for $\left\|p_{\boldsymbol{\Psi}}-p_{\boldsymbol{\Psi}, h}\right\|_{P}$ and the approximation properties of the $L^{2}$-orthogonal projector.

In practice, one can solve the problem with $\varphi \equiv 0$ and then post-process the pressure approximation according to Proposition 28. To check the theoretical results, we consider a numerical example based on the following manufactured solution on the unit square domain $\Omega:=(0,1)^{2}$ :

$u_{1}=-e^{x_{1}}\left(x_{2} \cos \left(x_{2}\right)+\sin \left(x_{2}\right)\right), \quad u_{2}=e^{x_{1}} x_{2} \sin \left(x_{2}\right), \quad p_{\Psi}=2 \exp \left(x_{1}\right) \sin \left(x_{2}\right)-C$, with $C$ such that $p_{\boldsymbol{\Psi}}$ has zero-mean on $\Omega, \boldsymbol{\Psi} \equiv \mathbf{0}$, and a potential

$$
\varphi=\chi \sin \left(2 \pi x_{1}\right) \sin \left(2 \pi x_{2}\right),
$$

where $\chi$ is a positive parameter that allows to adjust its intensity. In Figure 5 we compare the numerical results obtained with the modified right-hand side (62) to those obtained with a standard treatment (54) for the triangular and hexagonal mesh families depicted in Figure $3 \mathrm{a}$ and $3 \mathrm{~d}$, respectively. The results confirm that a standard treatment of the right-hand side does not yield satisfactory results.

\section{Appendix A. Mimicking the properties of the LOWEst-Order RAVIART-THOMAS SPACE ON GENERAL MESHES}

In the spirit of Section 3.1, it is possible to extend two classical properties of the lowest-order Raviart-Thomas space to general polygonal or polyhedral meshes, namely: (i) the (full) continuity of normal components of discrete functions at interfaces and (ii) the existence of an interpolator which preserves the mean value of the divergence inside each element. Since the construction as well as the proofs are similar to the ones presented in Section [3, only the main points are detailed. For a matching simplicial mesh, we introduce the broken polynomial space

$$
\mathbb{R} \mathbb{T}_{d}^{0}\left(\mathcal{K}_{h}\right):=\mathbb{P}_{d}^{0}\left(\mathcal{K}_{h}\right)^{d}+\boldsymbol{x} \mathbb{P}_{d}^{0}\left(\mathcal{K}_{h}\right)
$$




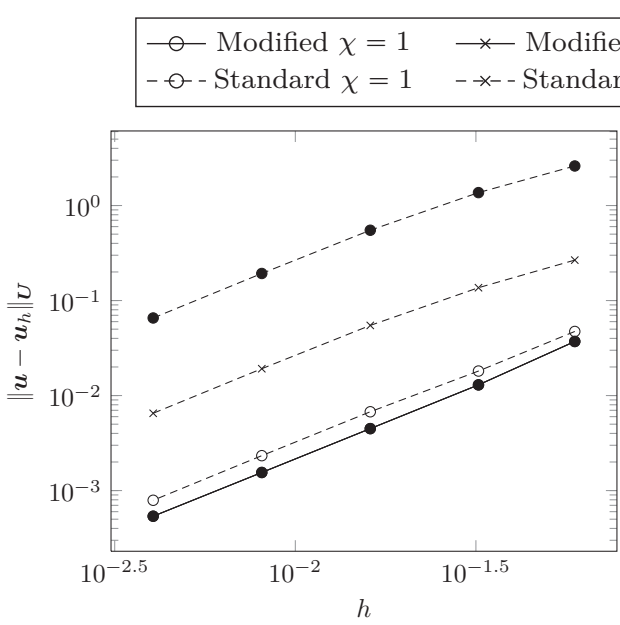

(A) Triangular mesh family

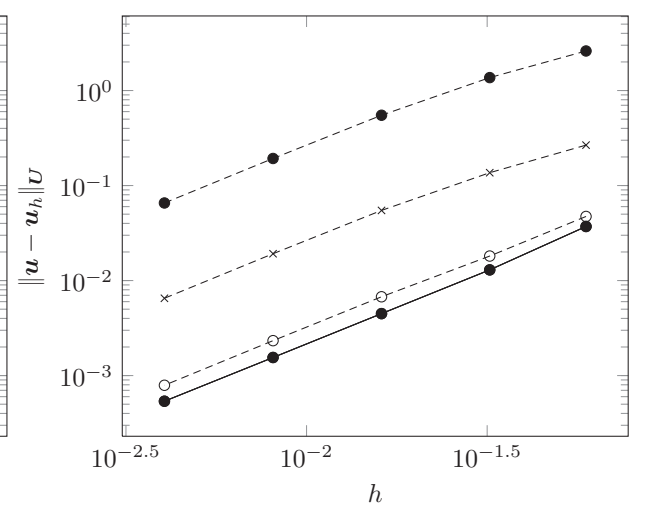

(B) Hexagonal mesh family

Figure 5. Effect of the treatment of the right-hand side (62) (Modified, solid lines) vs. (54) (Standard, dashed lines) when large irrotational volumetric forces are present. For a standard treatment of the right-hand side, the error on the velocity increases with $\chi$.

The standard lowest-order Raviart-Thomas space is the subspace of $\boldsymbol{H}$ (div; $\Omega$ ) of functions belonging to $\mathbb{R} \mathbb{T}_{d}^{0}\left(\mathcal{K}_{h}\right)$. To perform a similar construction on general polygonal or polyhedral meshes, we consider the following space of DOFs, composed of vector cell unknowns and scalar face unknowns associated to the normal component of the discrete vector field:

$$
\mathbb{V}_{h}:=\left\{\nabla_{h}=\left(\left(\boldsymbol{v}_{K} \in \mathbb{R}^{d}\right)_{K \in \mathcal{K}_{h}},\left(v_{F}^{n} \in \mathbb{R}\right)_{F \in \mathcal{F}_{\mathcal{K}_{h}}}\right)\right\} .
$$

As it is the case for the extension of the Crouzeix-Raviart space discussed in Section 3 cell unknowns are used to define a piecewise constant subgrid correction on the pyramidal submesh. The main difference with respect to the construction of Section 3 is that we now define an isotropic instead of a full gradient operator. More specifically, we introduce the operator $\mathfrak{G}_{h}: \mathbb{V}_{h} \rightarrow \mathbb{P}_{d}^{0}\left(\mathcal{P}_{h}\right)$ which realizes the mapping $\nabla_{h} \mapsto \mathfrak{G}_{h}\left(\nabla_{h}\right)$ with

$$
\mathfrak{G}_{h}\left(\mathbb{\nabla}_{h}\right)_{\mid K_{F}}=G_{K}\left(\mathbb{\vee}_{h}\right)+R_{K_{F}}\left(\nabla_{h}\right), \quad \forall K \in \mathcal{K}_{h}, \forall F \in \mathcal{F}_{K},
$$

where

$$
\begin{aligned}
G_{K}\left(\nabla_{h}\right) & :=\frac{1}{d|K|} \sum_{F \in \mathcal{F}_{K}}|F| v_{F}^{n} \boldsymbol{n}_{F} \cdot \boldsymbol{n}_{K, F}, \\
R_{K_{F}}\left(\nabla_{h}\right) & :=\frac{\eta}{d_{K, F}}\left(v_{F}^{n} \boldsymbol{n}_{F}-\boldsymbol{v}_{K}-G_{K}\left(\nabla_{h}\right)\left(\overline{\boldsymbol{x}}_{F}-\boldsymbol{x}_{K}\right)\right) \cdot \boldsymbol{n}_{K, F},
\end{aligned}
$$

and $\eta>0$ is a user-dependent parameter. Extending the definition (63), we introduce the reconstruction operator $\mathfrak{R}_{h}: \mathbb{V}_{h} \rightarrow \mathbb{R T}_{d}^{0}\left(\mathcal{P}_{h}\right)$ which realizes the mapping $\nabla_{h} \mapsto \mathfrak{R}_{h}\left(\nabla_{h}\right)$ with

$$
\mathfrak{R}_{h}\left(\mathbb{v}_{h}\right)_{\mid K_{F}}(\boldsymbol{x})=\boldsymbol{v}_{K}+\mathfrak{G}_{h}\left(\nabla_{h}\right)_{\mid K_{F}}\left(\boldsymbol{x}-\boldsymbol{x}_{K}\right), \quad \forall K_{F} \in \mathcal{P}_{h}, \forall \boldsymbol{x} \in K_{F} .
$$


Unlike (10), there holds for all $K \in \mathcal{K}_{h}, \boldsymbol{v}_{K}=\mathfrak{R}_{h}\left(\mathbb{\nabla}_{h}\right)\left(\boldsymbol{x}_{K}\right)$, i.e., the cell unknown can now be interpreted as the value of the reconstruction at the cell center. This is a consequence of selecting the cell center as a starting point in (65). We consider the discrete space

$$
\mathfrak{R T}\left(\mathcal{K}_{h}\right):=\mathfrak{R}_{h}\left(\mathbb{V}_{h}\right) .
$$

Lemma $30\left(\boldsymbol{H}(\operatorname{div} ; \Omega)\right.$-conformity). Assume $\eta=1$ in (64). Then, for all $\boldsymbol{v}_{h} \in$ $\mathfrak{R T}\left(\mathcal{K}_{h}\right)$ and all $F \in \mathcal{F}_{\mathcal{P}_{h}}^{\mathrm{i}}$, there holds for all $\boldsymbol{x} \in F$,

$$
\llbracket \boldsymbol{v}_{h} \rrbracket_{F}(\boldsymbol{x}) \cdot \boldsymbol{n}_{F}=0 .
$$

Proof. Let $\boldsymbol{v}_{h} \in \mathfrak{R T}\left(\mathcal{K}_{h}\right)$ with $\boldsymbol{v}_{h}=\boldsymbol{R}_{h}\left(\nabla_{h}\right), F \in \mathcal{F}_{\mathcal{P}_{h}}^{\mathrm{i}}$, and $\boldsymbol{x} \in F$. We distinguish two cases.

(i) $F \in \mathcal{F}_{\mathcal{K}_{h}}^{\mathrm{i}}$ is an interface of the primal mesh $\mathcal{K}_{h}$ such that $F \subset \partial K_{1} \cap \partial K_{2}$. For $i \in\{1,2\}$ let, for the sake of brevity, $G_{i}:=G_{K_{i}}\left(\nabla_{h}\right), R_{i}:=R_{K_{i F}}\left(\nabla_{h}\right), d_{i}:=$ $d_{K_{i}, F}\left(\boldsymbol{n}_{K_{i}, F} \cdot \boldsymbol{n}_{F}\right)$, and

$$
\alpha_{i}:=R_{i}\left(\boldsymbol{x}-\boldsymbol{x}_{K_{i}}\right) \cdot \boldsymbol{n}_{F}=R_{i} d_{i}=\eta\left(v_{F}^{n} \boldsymbol{n}_{F}-\boldsymbol{v}_{K_{i}}-G_{i}\left(\overline{\boldsymbol{x}}_{F}-\boldsymbol{x}_{K_{i}}\right)\right) \cdot \boldsymbol{n}_{F},
$$

where we have used the fact that $\boldsymbol{x} \in F$ to infer $\left(\boldsymbol{x}-\boldsymbol{x}_{K_{i}}\right) \cdot \boldsymbol{n}_{F}=d_{i}$ and the fact that $\boldsymbol{n}_{F}=\boldsymbol{n}_{K_{1}, F}=-\boldsymbol{n}_{K_{2}, F}$ to infer $\left(\boldsymbol{n}_{K_{i}, F} \cdot \boldsymbol{n}_{F}\right) \boldsymbol{n}_{K_{i}, F}=\boldsymbol{n}_{F}$. Algebraic manipulations yield

$$
\alpha_{1}-\alpha_{2}=-\eta\left[\left(\boldsymbol{v}_{K_{1}}-\boldsymbol{v}_{K_{2}}\right) \cdot \boldsymbol{n}_{F}+G_{1} d_{1}-G_{2} d_{2}\right] .
$$

Using the previous relation in the definition of the jump at $\boldsymbol{x} \in F$ it is inferred that

$$
\begin{aligned}
\llbracket \boldsymbol{v}_{h} \rrbracket_{F}(\boldsymbol{x}) \cdot \boldsymbol{n}_{F} & =\boldsymbol{v}_{h \mid K_{1 F}}(\boldsymbol{x}) \cdot \boldsymbol{n}_{F}-\boldsymbol{v}_{h \mid K_{2 F}}(\boldsymbol{x}) \cdot \boldsymbol{n}_{F} \\
& =\left(\boldsymbol{v}_{K_{1}}-\boldsymbol{v}_{K_{2}}\right) \cdot \boldsymbol{n}_{F}+G_{1} d_{1}-G_{2} d_{2}+\alpha_{1}-\alpha_{2} \\
& =(1-\eta)\left[\left(\boldsymbol{v}_{K_{1}}-\boldsymbol{v}_{K_{2}}\right) \cdot \boldsymbol{n}_{F}+G_{1} d_{1}-G_{2} d_{2}\right] .
\end{aligned}
$$

As a consequence, the jump vanishes provided $\eta=1$.

(ii) $F \in \mathcal{F}_{\mathcal{P}_{h}}^{\mathrm{i}} \backslash \mathcal{F}_{\mathcal{K}_{h}}^{\mathrm{i}}$ is a lateral pyramidal face such that there exist a unique element $K \in \mathcal{K}_{h}$ and two faces $F_{1}, F_{2} \in \mathcal{F}_{K}$ such that $F \subset \partial K_{F_{1}} \cap \partial K_{F_{2}}$ (cf. Figure 2a $)$. There holds, letting for the sake of brevity $R_{i}:=R_{K_{F_{i}}}\left(\nabla_{h}\right), i \in\{1,2\}$,

$$
\llbracket \boldsymbol{v}_{h} \rrbracket_{F}(\boldsymbol{x}) \cdot \boldsymbol{n}_{F}=\boldsymbol{v}_{h \mid K_{F_{1}}}(\boldsymbol{x}) \cdot \boldsymbol{n}_{F}-\boldsymbol{v}_{h \mid K_{F_{2}}}(\boldsymbol{x}) \cdot \boldsymbol{n}_{F}=\left(R_{1}-R_{2}\right)\left(\boldsymbol{x}-\boldsymbol{x}_{K}\right) \cdot \boldsymbol{n}_{F}=0,
$$

since $\left(\boldsymbol{x}-\boldsymbol{x}_{K}\right)$ and $\boldsymbol{n}_{F}$ are orthogonal by definition. This concludes the proof.

Remark 31 (Role of $\eta$ in the proof of Lemma 30). Unlike Lemma 8, the parameter $\eta$ is here used to enforce the continuity of the normal component across the interfaces of the primal mesh rather than across lateral pyramidal faces.

Let $\mathcal{I}_{h}^{\mathfrak{R T}}: H^{(\Omega)^{d}} \rightarrow \mathfrak{R T}\left(\mathcal{K}_{h}\right)$ be such that $\mathcal{I}_{h}^{\mathfrak{R T}}(\boldsymbol{v}):=\mathfrak{R}_{h}\left(\mathbb{\vee}_{h}\right)$ with

$$
\mathbb{V}_{h} \ni \nabla_{h}=\left(\left(\Pi_{h}^{1} \boldsymbol{v}\left(\boldsymbol{x}_{K}\right)\right)_{K \in \mathcal{K}_{h}},\left(\langle\boldsymbol{v}\rangle_{F} \cdot \boldsymbol{n}_{F}\right)_{F \in \mathcal{F}_{\mathcal{K}_{h}}}\right) .
$$

The following result summarizes the most relevant approximation properties of $\mathcal{I}_{h}^{\mathfrak{R}}$. The proof is omitted as it closely resembles that of Lemma 10.

Lemma 32 (Approximation in $\mathfrak{R T}\left(\mathcal{K}_{h}\right)$ ). For all $\eta>0$ in (64) and all $\boldsymbol{v} \in H^{(\Omega)^{d}}$ there holds with $\boldsymbol{v}_{h}:=\mathcal{I}_{h}^{\mathfrak{R T}}(\boldsymbol{v})$,

$$
D_{h}\left(\boldsymbol{v}_{h}\right)=\Pi_{h}^{0}(\boldsymbol{\nabla} \cdot \boldsymbol{v}) .
$$


Moreover, there exists a real $C>0$ independent of the meshsize such that, for all $h \in \mathcal{H}$, all $K \in \mathcal{K}_{h}$, and all $\boldsymbol{v} \in H^{(\Omega)^{d}} \cap \boldsymbol{H}^{1}\left(\operatorname{div} ; \mathcal{K}_{h}\right)$ with $\boldsymbol{v}_{h}:=\mathcal{I}_{h}^{\mathfrak{R T}}(\boldsymbol{v})$, there holds

$$
\left\|\boldsymbol{v}-\boldsymbol{v}_{h}\right\|_{L^{2}(K)^{d}}+\left\|\boldsymbol{\nabla} \cdot \boldsymbol{v}-D_{h}\left(\boldsymbol{v}_{h}\right)\right\|_{L^{2}(K)} \leqslant C h_{K}\left(|\boldsymbol{v}|_{H^{1}(K)^{d}}+|\boldsymbol{\nabla} \cdot \boldsymbol{v}|_{H^{1}(K)}\right) .
$$

Remark 33 (The matching simplicial case). When $\mathcal{K}_{h}$ is matching simplicial, in the spirit of Proposition 9, we can prove that the lowest-order Raviart-Thomas space is a subspace of $\mathfrak{R T}\left(\mathcal{K}_{h}\right)$. We also emphasize that the assumption $\eta=1$ in Lemma 30 is mandatory for the continuity of normal values at interfaces also in the matching simplicial case.

\section{REFERENCES}

[1] G. Allaire, Analyse Numérique et Optimisation, Les Éditions de l'École Polytechnique, Palaiseau, 2009.

[2] Douglas N. Arnold, Franco Brezzi, and Jim Douglas Jr., PEERS: a new mixed finite element for plane elasticity, Japan J. Appl. Math. 1 (1984), no. 2, 347-367, DOI 10.1007/BF03167064. MR.840802(87h:65189)

[3] Lourenco Beirão Da Veiga, A mimetic discretization method for linear elasticity, M2AN Math. Model. Numer. Anal. 44 (2010), no. 2, 231-250, DOI 10.1051/m2an/2010001. MR2655949(2011d:65317)

[4] L. Beirão da Veiga, F. Brezzi, and L. D. Marini, Virtual elements for linear elasticity problems, SIAM J. Numer. Anal. 51 (2013), no. 2, 794-812, DOI 10.1137/120874746. MR3033033

[5] L. Beirão da Veiga, V. Gyrya, K. Lipnikov, and G. Manzini, Mimetic finite difference method for the Stokes problem on polygonal meshes, J. Comput. Phys. 228 (2009), no. 19, 7215-7232, DOI 10.1016/j.jcp.2009.06.034. MR2568590(2010k:65229)

[6] L. Beirão da Veiga, K. Lipnikov, and G. Manzini, Error analysis for a mimetic discretization of the steady Stokes problem on polyhedral meshes, SIAM J. Numer. Anal. 48 (2010), no. 4, 1419-1443, DOI 10.1137/090757411. MR2684341 (2011m:65246)

[7] Susanne C. Brenner, Korn's inequalities for piecewise $H^{1}$ vector fields, Math. Comp. 73 (2004), no. 247, 1067-1087, DOI 10.1090/S0025-5718-03-01579-5. MR2047078(2005c:65096)

[8] Susanne C. Brenner and L. Ridgway Scott, The Mathematical Theory of Finite Element Methods, 3rd ed., Texts in Applied Mathematics, vol. 15, Springer, New York, 2008. MR2373954(2008m:65001)

[9] Susanne C. Brenner and Li-Yeng Sung, Linear finite element methods for planar linear elasticity, Math. Comp. 59 (1992), no. 200, 321-338, DOI 10.2307/2153060. MR1140646 (93a:73078)

[10] Franco Brezzi and Michel Fortin, Mixed and Hybrid Finite Element Methods, Springer Series in Computational Mathematics, vol. 15, Springer-Verlag, New York, 1991. MR 1115205 (92d:65187)

[11] Franco Brezzi, Konstantin Lipnikov, and Mikhail Shashkov, Convergence of the mimetic finite difference method for diffusion problems on polyhedral meshes, SIAM J. Numer. Anal. 43 (2005), no. 5, 1872-1896 (electronic), DOI 10.1137/040613950. MR2192322(2006j:65311)

[12] Franco Brezzi, Konstantin Lipnikov, Mikhail Shashkov, and Valeria Simoncini, A new discretization methodology for diffusion problems on generalized polyhedral meshes, Comput. Methods Appl. Mech. Engrg. 196 (2007), no. 37-40, 3682-3692, DOI 10.1016/j.cma.2006.10.028. MR 2339994(2008f:65102)

[13] Franco Brezzi, Konstantin Lipnikov, and Valeria Simoncini, A family of mimetic finite difference methods on polygonal and polyhedral meshes, Math. Models Methods Appl. Sci. 15 (2005), no. 10, 1533-1551, DOI 10.1142/S0218202505000832. MR2168945(2006i:65172)

[14] K. S. Chavan, B. P. Lamichhane, and B. I. Wohlmuth, Locking-free finite element methods for linear and nonlinear elasticity in $2 D$ and 3D, Comput. Methods Appl. Mech. Engrg., 196:4075-4086, 2007. 
[15] M. Crouzeix and P.-A. Raviart, Conforming and nonconforming finite element methods for solving the stationary Stokes equations. I, Rev. Française Automat. Informat. Recherche Opérationnelle Sér. Rouge 7 (1973), no. R-3, 33-75. MR0343661 (49 \#8401)

[16] Daniele A. Di Pietro, Cell centered Galerkin methods for diffusive problems, ESAIM Math. Model. Numer. Anal. 46 (2012), no. 1, 111-144, DOI 10.1051/m2an/2011016. MR2846369 (2012j:65397)

[17] D. A. Di Pietro and A. Ern, Mathematical Aspects of Discontinuous Galerkin Methods, Mathématiques \& Applications, vol. 69, Springer-Verlag, Berlin, 2012. MR2882148

[18] Daniele A. Di Pietro, Jean-Marc Gratien, and Christophe Prud'homme, A domain-specific embedded language in $C++$ for lowest-order discretizations of diffusive problems on general meshes, BIT 53 (2013), no. 1, 111-152, DOI 10.1007/s10543-012-0403-3. MR3029297

[19] Daniele A. Di Pietro and Serge Nicaise, A locking-free discontinuous Galerkin method for linear elasticity in locally nearly incompressible heterogeneous media, Appl. Numer. Math. 63 (2013), 105-116, DOI 10.1016/j.apnum.2012.09.009. MR2997905

[20] Jérôme Droniou and Robert Eymard, A mixed finite volume scheme for anisotropic diffusion problems on any grid, Numer. Math. 105 (2006), no. 1, 35-71, DOI 10.1007/s00211-006-00341. MR2257385 (2008d:65121)

[21] Jérôme Droniou and Robert Eymard, Study of the mixed finite volume method for Stokes and Navier-Stokes equations, Numer. Methods Partial Differential Equations 25 (2009), no. 1, 137-171, DOI 10.1002/num.20333. MR2473683(2009k:65208)

[22] Jérôme Droniou, Robert Eymard, Thierry Gallouët, and Raphaèle Herbin, A unified approach to mimetic finite difference, hybrid finite volume and mixed finite volume methods, Math. Models Methods Appl. Sci. 20 (2010), no. 2, 265-295, DOI 10.1142/S0218202510004222. MR 2649153(2011d:65318)

[23] Todd Dupont and Ridgway Scott, Polynomial approximation of functions in Sobolev spaces, Math. Comp. 34 (1980), no. 150, 441-463, DOI 10.2307/2006095. MR.559195(81h:65014)

[24] Alexandre Ern and Jean-Luc Guermond, Theory and Practice of Finite Elements, Applied Mathematical Sciences, vol. 159, Springer-Verlag, New York, 2004. MR2050138(2005d:65002)

[25] R. Eymard, T. Gallouët, and R. Herbin, Discretization of heterogeneous and anisotropic diffusion problems on general nonconforming meshes SUSHI: a scheme using stabilization and hybrid interfaces, IMA J. Numer. Anal. 30 (2010), no. 4, 1009-1043, DOI 10.1093/imanum/drn084. MR2727814(2012d:65246)

[26] Richard S. Falk, Nonconforming finite element methods for the equations of linear elasticity, Math. Comp. 57 (1991), no. 196, 529-550, DOI 10.2307/2938702. MR1094947 (92a:65290)

[27] Keith J. Galvin, Alexander Linke, Leo G. Rebholz, and Nicholas E. Wilson, Stabilizing poor mass conservation in incompressible flow problems with large irrotational forcing and application to thermal convection, Comput. Methods Appl. Mech. Engrg. 237/240 (2012), 166-176, DOI 10.1016/j.cma.2012.05.008. MR2947652

[28] Peter Hansbo and Mats G. Larson, Discontinuous Galerkin methods for incompressible and nearly incompressible elasticity by Nitsche's method, Comput. Methods Appl. Mech. Engrg. 191 (2002), no. 17-18, 1895-1908, DOI 10.1016/S0045-7825(01)00358-9. MR1886000 (2003j:74057)

[29] Peter Hansbo and Mats G. Larson, Discontinuous Galerkin and the Crouzeix-Raviart element: application to elasticity, M2AN Math. Model. Numer. Anal. 37 (2003), no. 1, 63-72, DOI 10.1051/m2an:2003020. MR.1972650(2004b:65184)

[30] Bishnu P. Lamichhane and Ernst P. Stephan, A symmetric mixed finite element method for nearly incompressible elasticity based on biorthogonal systems, Numer. Methods Partial Differential Equations 28 (2012), no. 4, 1336-1353, DOI 10.1002/num.20683. MR2914794

[31] Rolf Stenberg, A family of mixed finite elements for the elasticity problem, Numer. Math. 53 (1988), no. 5, 513-538, DOI 10.1007/BF01397550. MR954768 (89h:65192)

[32] Gilbert Strang, Variational crimes in the finite element method, The mathematical foundations of the finite element method with applications to partial differential equations (Proc. Sympos., Univ. Maryland, Baltimore, Md., 1972), Academic Press, New York, 1972, pp. 689710. MR0413554(54 \#1668)

[33] Michael Vogelius, An analysis of the p-version of the finite element method for nearly incompressible materials. Uniformly valid, optimal error estimates, Numer. Math. 41 (1983), no. 1, 39-53, DOI 10.1007/BF01396304. MR696549(85f:65113b) 
[34] Martin Vohralík and Barbara I. Wohlmuth, From face to element unknowns by local static condensation with application to nonconforming finite elements, Comput. Methods Appl. Mech. Engrg. 253 (2013), 517-529, DOI 10.1016/j.cma.2012.08.013. MR3002809

[35] Martin Vohralík and Barbara I. Wohlmuth, Mixed finite element methods: implementation with one unknown per element, local flux expressions, positivity, polygonal meshes, and relations to other methods, Math. Models Methods Appl. Sci. 23 (2013), no. 5, 803-838, DOI 10.1142/S0218202512500613. MR3028542

Université Montpellier 2, I3M, 34057 Montpellier CEDEX 5, France

E-mail address: daniele.di-pietro@univ-montp2.fr

IFP Énergies nouvelles, Department of Applied Mathematics, 1 \& 4 avenue de BoisPréau, 92852 Rueil-Malmaison CEDEX, France

E-mail address: simon.lemaire87@gmail.com 